\title{
Transport properties in the $d$-density-wave state in an external magnetic field: The Wiedemann-Franz law
}

\author{
S.G. Sharapov ${ }^{1}$ * V.P. Gusynin ${ }^{1,2}$ 母 and H. Beck团 \\ ${ }^{1}$ Institut de Physique, Université de Neuchâtel, 2000 Neuchâtel, Switzerland \\ ${ }^{2}$ Bogolyubov Institute for Theoretical Physics, Metrologicheskaya Str. 14-b, Kiev, 03143, Ukraine
}

(Dated: November 5, 2018)

\begin{abstract}
We derive the electrical and thermal conductivities of the $d$-density-wave state in an external magnetic field $B$ in the low-temperature regime and in the presence of impurities. We show that in the zero-temperature limit, $T \rightarrow 0$, the Wiedemann-Franz (WF) law remains intact irrespectively of the value of the applied field and chemical potential $\mu$ as soon as there is scattering from impurities. For finite $T \lesssim|\mu|$ the WF law violation is possible and it is enhanced by the external field. Depending on the relative values of $B$ and $T$ the electrical conduction may dominate the heat conduction and vice versa. For $\mu \gg T$ the WF is restored even in the presence of the external field.
\end{abstract}

PACS numbers: 71.10.-w, 74.25.Fy, 74.72.-h, 11.10.Wx

\section{INTRODUCTION}

A recent experiment of Hill et al. [1] that measured electrical and thermal conductivities of the optimally doped copper oxide superconductor $\mathrm{Pr}_{2-x} \mathrm{Ce}_{x} \mathrm{CuO}_{4}$ (PCCO) in its normal state found striking deviations from the WiedemannFranz (WF) law. The electron-doped PCCO compound with $T_{c} \simeq 20 \mathrm{~K}$ near the optimal $x=0.15$ doping is the analog of the hole doped material $\mathrm{La}_{2-x} \mathrm{Sr}_{x} \mathrm{CuO}_{4-y}$ (LSCO), which is also optimally doped at approximately $x=0.15$ with a maximum $T_{c} \simeq 40 \mathrm{~K}$. Lower $T_{c}$ in PCCO makes it particularly convenient from a technical point of view since an external magnetic field is necessary to destroy superconductivity to study its low temperature transport properties in the normal state. While for LSCO the upper critical field is $\simeq 50 \mathrm{~T}$, in the optimally doped PCCO the superconductivity is already destroyed at $8 \mathrm{~T} \mathrm{[1]}$. The choice of overdoped compounds that can be driven into the normal state by a relatively small magnetic field $\sim 13 \mathrm{~T}$ is wider and the hole overdoped system $\mathrm{Tl}_{2} \mathrm{Ba}_{2} \mathrm{CuO}_{6+\delta}(\mathrm{Tl}-2201)$ with $T_{c}=15 \mathrm{~K}$ was studied very recently by Proust et al. [2]. They verified that in the overdoped Tl-2201 WF law holds perfectly.

The WF law is one of the basic properties of a Fermi liquid, reflecting the fact that the ability of a quasiparticle to transport heat is the same as its ability to transport charge, provided it cannot lose energy through collisions. The WF law states that the ratio of the heat conductivity $\kappa$ to the electrical conductivity $\sigma$ of a metal is a universal constant:

$$
L_{0} \equiv \frac{\kappa}{\sigma T}=\frac{\pi^{2}}{3}\left(\frac{k_{B}}{e}\right)^{2}
$$

where $k_{B}$ is the Boltzmann constant, $e$ is electron charge, and $L_{0}=2.45 \times 10^{-8} \mathrm{~W} \Omega \mathrm{K}^{-2}$ is Sommerfeld's value for the Lorenz ratio $L \equiv \kappa /(\sigma T)$. To be more precise, one should also specify the temperature range where the WF law holds. Strictly speaking this law is proven only in the limit $T \rightarrow 0$ and for a small concentration of impurities [3]. In a less strict sense it is often implied that the WF law is valid for $k_{B} T \ll \mu$, where $\mu$ is chemical potential. In this case one can provide some qualitative arguments $[4]$ that if the scattering from impurities does not strongly depend on the energy the WF will still be valid. Moreover, for $k_{B} T \ll \mu$ and elastic electron scattering these arguments can also be extended to the case of a strong $\left(\hbar \omega_{L} \gg k_{B} T, \hbar \omega_{L} \gg \Gamma\right)$ quantizing magnetic field [5], where $\hbar \omega_{L}$ is the distance between Landau levels and $\Gamma$ is the impurity scattering rate. Thus, if these conditions are fulfilled, any violation of the WF law would indicate that there is a breakdown of the picture based on the Fermi liquid theory.

One of the possible theoretical interpretations of the WF law breakdown is that the quasiparticle fractionalizes into separate spin and charge. This separation can be investigated using various models and approaches, so here we mention only the most recent studies done in the context of the WF law violation in cuprates. Specifically, the WF law

\footnotetext{
*Electronic address: Sergei.Sharapov@unine.ch

${ }^{\dagger}$ Electronic address: vgusynin@bitp.kiev.ua

${ }^{\ddagger}$ Electronic address: Hans.Beck@unine.ch URL: http://www.unine.ch/phys/theocond/
} 
violation has been studied for a large- $N$ limit of the $t-J$ model on the square lattice 6 ]. Another examination of the WF law was done by Yang and Nayak (YN) [7] and also by Kim and Carbotte (KC) [] within the phenomenological $d$-density-wave (DDW) picture.

The DDW scenario proposed in Ref. [9] is based on the assumption that the pseudogap phenomenon [10] in high- $T_{c}$ cuprates is the result of the development of another order parameter called the DDW order [11] that has $d$-wave symmetry:

$$
\left\langle c_{s}^{\dagger}(t, \mathbf{k}+\mathbf{Q}) c_{s^{\prime}}(t, \mathbf{k})\right\rangle=i \Phi_{\mathbf{Q}} f(\mathbf{k}) \delta_{s s^{\prime}}
$$

where $s, s^{\prime}= \pm$ is the spin index, $f(\mathbf{k})=\cos k_{x} a-\cos k_{y} a, \mathbf{Q}=(\pi / a, \pi / a)$, and $a$ is the lattice constant. In comparison to the $d$-wave superconducting order the DDW order parameter appears to be rather exotic because it breaks the time reversal, translation by one lattice spacing and rotation by $\pi / 2$ symmetries, but respects any combination of two of these. The underlying reason for this breaking is that there are countercirculating currents in the DDW ground state. The schematic phase diagram for the DDW pseudogap scenario is shown in Fig. 11 For a hole doping larger than the critical value $x_{c} \approx 0.19$ [12], the DDW order is presumed to disappear, whereas for the underdoped system DDW order coexists with $d$-wave superconductivity ( $d \mathrm{SC}$ ).

One of the unusual features of the DDW state is that for a half-filled system the chemical potential of the nodal quasiparticles participating in the electrical and thermal transport can be small or even zero, i.e., $|\mu|<k_{B} T$, which violates the usual conditions of the WF law validity. Indeed, exactly in the limit $\mu=0$ the WF law is strongly violated in the extremely clean limit [7]. There is no WF law violation in the $T=0$ limit for finite $\mu$ and/or $\Gamma$ [7, 8]. For finite temperatures the WF violation depends on the impurity scattering [8] : in the Born limit (for a constant impurity scattering rate) there is no change in the WF law, but in the unitary limit for the frequency dependent scattering rate the WF law is violated, but only for $|\mu|$ smaller that the DDW gap. When $\mu$ is increased sufficiently, the Lorenz number becomes approximately equal to its conventional value and its temperature dependence is small.

While in general the validity of the DDW pseudogap scenario is still questionable, it is important to scrutinize all its theoretical consequences. One of the opportunities is to study possible WF law violations using the DDW model, so that these results can be compared with the experimental results of Refs. 11, 2]. Although this investigation to a large extent had already been done by YN [7] and KC [8], both these papers do not consider the presence of the external magnetic field which is an essential ingredient of the experiments [1, 2]. Indeed, in these experiments the DDW state appears in the magnetic field in the underdoped regime at low $T$ when the superconductivity is destroyed.

In this paper we study the WF law for the DDW model including the external magnetic field for a constant impurity scattering rate, paying special attention to the regime $|\mu| \lesssim k_{B} T$ where the violation of the WF law is expected. Although there are some formal similarities between the DDW and $d$-wave superconducting states, the present problem is much simpler because there are no vortices and no superflows surrounding them in the DDW state. The $U(1)$ symmetry in the DDW state also remains unbroken, so that the electromagnetic field enters into the effective low-energy theory in the same way as in $\mathrm{QED}_{2+1}$. Thus we avoid the famous problem of Dirac Landau-level mixing (see Refs. 13, 14]).

We begin by presenting in Sec. Пthe model DDW Hamiltonian and rewriting it using two-component spinors. Since there is a discrepancy between the expressions for the electrical current used by YN [7] and KC [8], we also derive the electrical current operator and show that in the nodal approximation it reduces to the current of Ref. [7]. Then we obtain the low-energy effective action (Lagrangian) for the nodal quasiparticles in the presence of an external electromagnetic field, consider its discrete symmetries, and rewrite the Lagrangian in the Dirac form. In Sec. IIII we consider the Green's function of nodal quasiparticles in an applied field (the calculational details are given in Appendix A. In Appendix B using this Green's function, we derive a generalized polarization bubble that can be applied to the calculation of electrical and thermal conductivities. In Secs. IV and $\mathbb{\nabla}$ we obtain general expressions for electrical and thermal conductivities in the external field. These general expressions are further analyzed in detail by analytical and numerical methods. In Sec. $\mathrm{VI}$ we investigate the implications for the WF law that follow from the results presented in the previous sections. Conclusions are discussed in Sec. [VII where we give a concise summary of the obtained results and compare them with experiment. Some useful integrals are given in Appendix [ ]

\section{II. $d$-DENSITY WAVE HAMILTONIAN AND ITS EFFECTIVE LOW ENERGY FORM}

We start with the mean-field Hamiltonian for the DDW state 7, 11, 15

$$
H^{\mathrm{DDW}}=\int_{\mathrm{BZ}} \frac{d^{2} k}{(2 \pi)^{2}}\left[(\varepsilon(\mathbf{k})-\mu) c_{s}^{\dagger}(\mathbf{k}) c_{s}(\mathbf{k})+i D(\mathbf{k}) c_{s}^{\dagger}(\mathbf{k}) c_{s}(\mathbf{k}+\mathbf{Q})\right]
$$

where the single particle energy is $\varepsilon(\mathbf{k})=-2 t\left(\cos k_{x} a+\cos k_{y} a\right)-4 t^{\prime} \cos k_{x} a \cos k_{y} a$ with $t, t^{\prime}$ being the hopping parameters, $\mu$ is the chemical potential, $D(\mathbf{k})=\frac{D_{0}}{2}\left(\cos k_{x} a-\cos k_{y} a\right)$ is the $d$-density-wave gap and $\mathbf{Q}=(\pi / a, \pi / a)$ 
is the wave vector at which the density-wave ordering takes place. The integral is over the full Brillouin zone. Throughout the paper $\hbar=k_{B}=c=1$ units are chosen, unless stated explicitly otherwise.

Due to the presence of the factor $i$ in the second term of the Hamiltonian (2.1), it breaks time-reversal symmetry, where the time-reversal operation is defined as 16$]$

$$
c_{s}(\mathbf{k}) \rightarrow \mathcal{T} c_{s}(\mathbf{k}) \mathcal{T}^{-1}=i s c_{-s}(-\mathbf{k}), \quad c_{s}^{\dagger}(\mathbf{k}) \rightarrow \mathcal{T} c_{s}^{\dagger}(\mathbf{k}) \mathcal{T}^{-1}=-i s c_{-s}^{\dagger}(-\mathbf{k})
$$

and the time-reversal operator $\mathcal{T}$ is unitary and antilinear. Nevertheless the Hamiltonian (2.1) preserves a combined symmetry of the time reversal and a translation by one lattice spacing $\tilde{\mathcal{T}}=\mathcal{T} T_{\mathbf{a}}$ :

$$
c_{s}(\mathbf{k}) \rightarrow \tilde{\mathcal{T}} c_{s}(\mathbf{k}) \tilde{\mathcal{T}}^{-1}=i e^{-i \mathbf{k a}} c_{-s}(-\mathbf{k})
$$

Since the order parameter also breaks translational symmetry by one lattice spacing, it is convenient to halve the Brillouin zone as shown in Fig. 2 and form a two-component electron operator

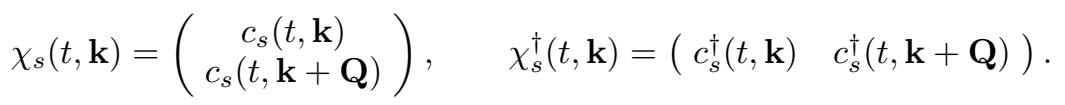

Then the mean-field Hamiltonian (2.1) in terms of $\chi$ becomes

$$
H^{\mathrm{DDW}}=\int_{\mathrm{RBZ}} \frac{d^{2} k}{(2 \pi)^{2}} \chi_{s}^{\dagger}(t, \mathbf{k})\left[\frac{1}{2}[\varepsilon(\mathbf{k})+\varepsilon(\mathbf{k}+\mathbf{Q})]-\mu+\frac{1}{2}[\varepsilon(\mathbf{k})-\varepsilon(\mathbf{k}+\mathbf{Q})] \sigma_{3}-D(\mathbf{k}) \sigma_{2}\right] \chi_{s}(t, \mathbf{k}),
$$

where $\sigma_{i}$ are Pauli matrices and the integral is over the reduced Brillouin zone (RBZ). The symmetry operation (2.3), for example, is

$$
\chi_{s}(t, \mathbf{k}) \rightarrow \tilde{\mathcal{T}} \chi_{s}(t, \mathbf{k}) \tilde{\mathcal{T}}^{-1}=-e^{-i \mathbf{k a}} \sigma_{2} \chi_{-s}(-t,-\mathbf{k}-\mathbf{Q})
$$

In what follows we will consider the more simple case $t^{\prime}=0$, so that after employing the nesting property $\varepsilon(\mathbf{k}+\mathbf{Q})=$ $-\varepsilon(\mathbf{k})$ the Hamiltonian (2.5) takes a simple form

$$
H^{\mathrm{DDW}}=\int_{\mathrm{RBZ}} \frac{d^{2} k}{(2 \pi)^{2}} \chi_{s}^{\dagger}(t, \mathbf{k})\left[H_{0}(\mathbf{k})-\mu\right] \chi_{s}(t, \mathbf{k})
$$

where

$$
H_{0}(\mathbf{k})=\varepsilon(\mathbf{k}) \sigma_{3}-D(\mathbf{k}) \sigma_{2}
$$

\section{A. Electrical and energy current operators}

As we already mentioned the expressions for the electrical current used in Refs. 7] and [8] are different. Although quantitatively this difference for high-temperature superconductors is expected to be rather small [see after Eq. (2.21)], the extra term in the current present in Ref. 7] is of qualitative importance, so we need to discuss its origin.

To find out electrical current operator $\mathbf{j}$ for Hamiltonian (2.1) we apply the method similar to that of Refs. 17] and [8], where it was used to determine the heat current in the $d$-wave superconducting and DDW states, respectively. The idea of the derivation is to make the expression for the current operator compatible with the charge conservation law,

$$
\frac{\partial \rho(t, \mathbf{r})}{\partial t}+\nabla \cdot \mathbf{j}(\mathbf{t}, \mathbf{r})=0
$$

and with the equations of motion for field operators,

$$
i \frac{\partial c_{s}^{\dagger}(\mathbf{k})}{\partial t}=\left[c_{s}^{\dagger}(\mathbf{k}), H\right]=-c_{s}^{\dagger}(\mathbf{k})[\varepsilon(\mathbf{k})-\mu]-c_{s}^{\dagger}(\mathbf{k}-\mathbf{Q}) i D(\mathbf{k}-\mathbf{Q})
$$

Writing Eq. (2.9) in the momentum space, one obtains

$$
\frac{\partial \rho(t, \mathbf{q})}{\partial t}+i \mathbf{q} \cdot \mathbf{j}(t, \mathbf{q})=0
$$


where the Fourier transform of the charge density is

$$
\rho(t, \mathbf{q})=e \int_{\mathrm{BZ}} \frac{d^{2} k}{(2 \pi)^{2}} c_{s}^{\dagger}(\mathbf{k}) c_{s}(\mathbf{k}+\mathbf{q})
$$

For its derivative we obtain

$$
\frac{\partial \rho(t, \mathbf{q})}{\partial t}=-i e \int_{\mathrm{BZ}} \frac{d^{2} k}{(2 \pi)^{2}}\left[c_{s}^{\dagger}(\mathbf{k}) c_{s}(\mathbf{k}+\mathbf{q}) \mathbf{q} \frac{\partial \varepsilon(\mathbf{k})}{\partial \mathbf{k}}+c_{s}^{\dagger}(\mathbf{k}) c_{s}(\mathbf{k}+\mathbf{q}+\mathbf{Q}) i \mathbf{q} \frac{\partial D(\mathbf{k})}{\partial \mathbf{k}}\right], \quad \mathbf{q} \rightarrow 0
$$

so that the electrical current is

$$
\begin{aligned}
\mathbf{j}(t, \mathbf{0}) & =e \int_{\mathrm{BZ}} \frac{d^{2} k}{(2 \pi)^{2}}\left[c_{s}^{\dagger}(\mathbf{k}) c_{s}(\mathbf{k}) \frac{\partial \varepsilon(\mathbf{k})}{\partial \mathbf{k}}+c_{s}^{\dagger}(\mathbf{k}) c_{s}(\mathbf{k}+\mathbf{Q}) i \frac{\partial D(\mathbf{k})}{\partial \mathbf{k}}\right] \\
& =e \int_{\mathrm{RBZ}} \frac{d^{2} k}{(2 \pi)^{2}} \chi_{s}^{\dagger}(\mathbf{k}) \mathbf{V}(\mathbf{k}) \chi_{s}(\mathbf{k}),
\end{aligned}
$$

where we introduced the generalized velocity operator that depends of the Fermi, $\mathbf{v}_{F}$, and gap, $\mathbf{v}_{D}$, velocities

$$
\mathbf{V}(\mathbf{k})=\mathbf{v}_{F}(\mathbf{k}) \sigma_{3}+\mathbf{v}_{D}(\mathbf{k}) \sigma_{2}, \quad \mathbf{v}_{F}(\mathbf{k}) \equiv \frac{\partial \epsilon(\mathbf{k})}{\partial \mathbf{k}}, \quad \mathbf{v}_{D}(\mathbf{k}) \equiv-\frac{\partial D(\mathbf{k})}{\partial \mathbf{k}}
$$

In the last equality in Eq. (2.14) we used the two-component form and relied on the nesting property $\varepsilon(\mathbf{k}+\mathbf{Q})=-\varepsilon(\mathbf{k})$ and $D(\mathbf{k}+\mathbf{Q})=-D(\mathbf{k})$.

This form of the electrical current operator reduces to the current used by YN [7] after the nodal approximation is made. The last term of Eq. (2.14), $\sim \mathbf{v}_{D}$, is due to the fact that the DDW gap in the mean-field Hamiltonian (2.1) is k-dependent. The expression for the electrical current used by KC contains only one term $\sim \mathbf{v}_{F}$. In this case, calculating electrical conductivity in the bare bubble approximation is not consistent with charge-current conservation, since such a bare vertex does not satisfy the Ward identity when quasiparticle self-energy has a nontrivial momentum dependence. Instead one should use a vertex that complies with the Ward identity.

The derivation of the energy current operator from the Hamiltonian (2.1) is quite similar to the derivation of the electrical current operator (2.14), so here we only outline the main steps. This current is calculated from the corresponding continuity equation for the energy density and the equations of motion (2.10) (we set there $\mu=0$ because the energy density is considered) that give

$$
\mathbf{j}^{E}(t, \mathbf{0})=\int_{\mathrm{BZ}} \frac{d^{2} k}{(2 \pi)^{2}}\left[\varepsilon(\mathbf{k}) \mathbf{v}_{F}(\mathbf{k})+D(\mathbf{k}) \mathbf{v}_{D}(\mathbf{k})\right] c_{s}^{\dagger}(\mathbf{k}) c_{s}(\mathbf{k})
$$

or in the spinor form

$$
\mathbf{j}^{E}(t, \mathbf{0})=\frac{1}{2} \int_{\mathrm{RBZ}} \frac{d^{2} k}{(2 \pi)^{2}} \chi^{\dagger}(t, \mathbf{k})\left\{H_{0}(\mathbf{k}), \mathbf{V}(\mathbf{k})\right\} \chi(t, \mathbf{k}) .
$$

Then using again the equations of motion we finally get

$$
\mathbf{j}^{E}(\Omega, \mathbf{0})=\int_{\mathrm{RBZ}} \frac{d^{2} k}{(2 \pi)^{2}} \int d \omega\left(\omega+\frac{\Omega}{2}\right) \chi^{\dagger}(\omega, \mathbf{k}) \mathbf{V}(\mathbf{k}) \chi(\omega+\Omega, \mathbf{k}) .
$$

One can easily see that Eq. (2.18) agrees with the corresponding energy current obtained in Ref. [8].

\section{B. Effective low-energy nodal action}

The action $S$ corresponding to the Hamiltonian (2.7) is

$$
\begin{aligned}
S & =-\int_{0}^{\beta} d \tau\left[\int d^{2} r \chi_{s}^{\dagger}(\tau, \mathbf{r}) \partial_{\tau} \chi_{s}(\tau, \mathbf{r})+H^{\mathrm{DDW}}(\tau)\right] \\
& =-\int_{0}^{\beta} d \tau \int_{\mathrm{RBZ}} \frac{d^{2} k}{(2 \pi)^{2}} \chi_{s}^{\dagger}(\tau, \mathbf{k})\left[\partial_{\tau}-\mu+\varepsilon(\mathbf{k}) \sigma_{3}-D(\mathbf{k}) \sigma_{2}\right] \chi_{s}(\tau, \mathbf{k}),
\end{aligned}
$$


where $\tau$ is the imaginary time. Hence the Green's function of the DDW state reads

$$
G(i \omega, \mathbf{k})=\frac{(i \omega+\mu) \hat{I}+\varepsilon(\mathbf{k}) \sigma_{3}-D(\mathbf{k}) \sigma_{2}}{(i \omega+\mu)^{2}-E^{2}(\mathbf{k})}, \quad E(\mathbf{k})=\sqrt{\varepsilon^{2}(\mathbf{k})+D^{2}(\mathbf{k})}
$$

where $i \omega=i(2 n+1) \pi T$ is fermionic (odd) Matsubara frequency.

Linearizing the spectrum about the four nodes $\mathbf{N}=( \pm \pi / 2 a, \pm \pi / 2 a)$ at half-filling $(\mu=0)$, one obtains the Green's function (GF) for a given node (we choose the local nodal coordinate systems as shown in Fig. 21)

$$
G_{\text {node }}(i \omega, \mathbf{k})=\frac{(i \omega+\mu) \hat{I}+v_{F} k_{x} \sigma_{3}+v_{D} k_{y} \sigma_{2}}{(i \omega+\mu)^{2}-E^{2}(\mathbf{k})}, \quad E(\mathbf{k})=\sqrt{v_{F}^{2} k_{x}^{2}+v_{D}^{2} k_{y}^{2}}
$$

where the Fermi velocity for half-filling $v_{F}=|\partial \varepsilon(\mathbf{k}) / \partial \mathbf{k}|_{\mathbf{k}=\mathbf{N}} \mid=2 \sqrt{2} t a$ and the DDW gap velocity $v_{D}=$ $|\partial D(\mathbf{k}) / \partial \mathbf{k}|_{\mathbf{k}=\mathbf{N}} \mid=\frac{1}{\sqrt{2}} D_{0} a$. It is important to stress that this way of linearization is different from the nodal approximation used to describe $d \mathrm{SC}$ [17] when the expansion is done around the nodal ("Dirac") points defined as an intersection of the Fermi surface $\varepsilon(\mathbf{k})-\mu=0$ and the nodal lines $D(\mathbf{k})=0$. For the DDW case one always expands around half-filling $\varepsilon(\mathbf{k})=0$ and $D(\mathbf{k})=0$, so that for nonzero $\mu$ the nodes transform into small pockets (see Fig. 2) which are cross sections of the "Dirac cone". Thus only at exactly half-filling is there a correspondence between DDW and $d \mathrm{SC}$ cases. There are the following estimates [18] of the model parameters, $t=300 \mathrm{meV}$ and $D_{0}=50 \mathrm{meV}$, which give the ratio $v_{F} / v_{D}=24$. This shows that the second term of the electrical current (2.14), which is proportional to $v_{D}$, is indeed small. The value of the chemical potential corresponding to a hole doping of $10 \%$ is [18] $\mu=0.91 t$.

The linearized Dirac action that corresponds to the Green's function (2.21) can be written as

$$
S_{\text {node }}=-\int_{0}^{\beta} d \tau \int d^{2} r \chi_{s}^{\dagger}(\tau, \mathbf{r})\left[\hat{I}\left(\partial_{\tau}-\mu\right)-v_{F} \sigma_{3} i \partial_{x}-v_{D} \sigma_{2} i \partial_{y}\right] \chi_{s}(\tau, \mathbf{r}) .
$$

Dealing with Eqs. (2.21) and (2.22), one should not forget that all physical quantities involve the summation over the 4 nodes present in the original Green's function (2.20). In what follows we will use the nodal action (2.22) instead of the original DDW Hamiltonian (2.1). This approximation is well justified for $T \ll D_{0}$. As we will see below, this approximation and the fact that the electromagnetic field enters the theory through the minimal coupling prescription will allow us to apply the results of $\mathrm{QED}_{2+1}$.

\section{Discrete symmetries of the nodal Lagrangian}

Since the action (2.22) does not directly correspond to the common $\mathrm{QED}_{2+1}$ form used in the literature [19] (see also Ref. [20]), we present below the transformations for the discrete symmetries. Let us consider only one node and suppress the spin index $s$, so that in real time $t=-i \tau$ the action $S$ reads

$$
S_{\text {node }}=i \int d t \int d^{2} r \mathcal{L}
$$

with

$$
\mathcal{L}=\chi^{\dagger}(t, \mathbf{r})\left[i \hat{I}\left(\partial_{t}-i e A_{0}(t, \mathbf{r})\right)+\mu+i v_{F} \sigma_{3}\left(\partial_{x}-i \frac{e}{c} A_{1}(t, \mathbf{r})\right)+i v_{D} \sigma_{2}\left(\partial_{y}-i \frac{e}{c} A_{2}(t, \mathbf{r})\right)\right] \chi(t, \mathbf{r}),
$$

where $A_{\nu}(t, \mathbf{r}), \nu=0,1,2$ is the electromagnetic field vector potential. This is a highly nontrivial fact in that the vector potential can be inserted in Eq. (2.24) using the minimal coupling prescription of QED. Only due to this fact can one apply the results of $\mathrm{QED}_{2+1}$ for the description of the DDW state. Moreover, this is only correct for the DDW state [7] but not for the $d$ SC state, where the last term of the Lagrangian (2.24) does not couple with the electromagnetic field. It has to be admitted that the way of inserting the electromagnetic field is not just related to the nodal approximation and can be traced back to the expression (2.14) for the electrical current that can only be obtained when the vector potential enters both $\varepsilon(\mathbf{k})$ and $D(\mathbf{k})$ terms in Eq. (2.1). Finally we note that the symmetries of the Lagrangian have to be analyzed in real time where one can distinguish time and spatial coordinates. 


\section{1. $\quad$ Parity}

In $2+1$ dimensions, parity corresponds to inverting one axis (since the inversion of both axes would be rotation by $\pi): P \mathbf{r}=\mathbf{r}^{\prime}$, where $\mathbf{r}=(x, y)$ and $\mathbf{r}^{\prime}=(x,-y)$. The corresponding operation on the two-component spinor and on the gauge field is

$$
\begin{aligned}
& \chi(\tau, \mathbf{r}) \rightarrow \mathcal{P} \chi(\tau, \mathbf{r}) \mathcal{P}^{-1}=\sigma_{3} \chi\left(\tau, r^{\prime}\right), \quad \chi^{\dagger}(t, \mathbf{r}) \rightarrow \mathcal{P} \chi^{\dagger}(t, \mathbf{r}) \mathcal{P}^{-1}=\chi^{\dagger}\left(t, \mathrm{r}^{\prime}\right) \sigma_{3}, \\
& A_{0}(t, \mathbf{r}) \rightarrow \mathcal{P} A_{0}(t, \mathbf{r}) \mathcal{P}^{-1}=A_{0}\left(t, \mathbf{r}^{\prime}\right), \quad A_{1}(t, \mathbf{r}) \rightarrow \mathcal{P} A_{1}(t, \mathbf{r}) \mathcal{P}^{-1}=A_{1}\left(t, \mathbf{r}^{\prime}\right), \quad A_{2}(t, \mathbf{r}) \rightarrow \mathcal{P} A_{2}(t, \mathbf{r}) \mathcal{P}^{-1}=-A_{2}\left(t, \mathbf{r}^{\prime}\right) .
\end{aligned}
$$

One can verify that the Lagrangian (2.24) is invariant under this parity transformation while possible mass terms with $\sigma_{1}$ and $\sigma_{2}$ break parity:

$$
\begin{aligned}
& \chi^{\dagger}(t, \mathbf{r}) \sigma_{1} \chi(t, \mathbf{r}) \rightarrow \mathcal{P} \chi^{\dagger}(t, \mathbf{r}) \sigma_{1} \chi(t, \mathbf{r}) \mathcal{P}^{-1}=-\chi^{\dagger}\left(t, \mathbf{r}^{\prime}\right) \sigma_{1} \chi\left(t, \mathbf{r}^{\prime}\right) ; \\
& \chi^{\dagger}(t, \mathbf{r}) \sigma_{2} \chi(t, \mathbf{r}) \rightarrow \mathcal{P} \chi^{\dagger}(t, \mathbf{r}) \sigma_{2} \chi(t, \mathbf{r}) \mathcal{P}^{-1}=-\chi^{\dagger}\left(t, \mathbf{r}^{\prime}\right) \sigma_{2} \chi\left(t, \mathbf{r}^{\prime}\right) .
\end{aligned}
$$

\section{Charge conjugation}

Charge conjugation,

$$
\begin{array}{ll}
\chi(t, \mathbf{r}) \rightarrow \mathcal{C} \chi(t, \mathbf{r}) \mathcal{C}^{-1}=\sigma_{3}\left[\chi^{\dagger}(t, \mathbf{r})\right]^{T}, & \chi^{\dagger}(t, \mathbf{r}) \rightarrow \mathcal{C} \chi^{\dagger}(t, \mathbf{r}) \mathcal{C}^{-1}=[\chi(t, \mathbf{r})]^{T} \sigma_{3}, \\
A_{\nu}(t, \mathbf{r}) \rightarrow \mathcal{C} A_{\nu}(t, \mathbf{r}) \mathcal{C}^{-1}=-A_{\nu}(t, \mathbf{r}), & \nu=0,1,2
\end{array}
$$

leaves the equations of motion invariant. It is easy to check that the Lagrangian (2.24) is invariant under this transformation only at half-filling $(\mu=0)$ while away from half-filling the term with the chemical potential breaks $C$

$$
\mu \chi^{\dagger}(t, \mathbf{r}) \hat{I} \chi(t, \mathbf{r}) \rightarrow \mu \mathcal{C} \chi^{\dagger}(t, \mathbf{r}) \hat{I} \chi(t, \mathbf{r}) \mathcal{C}^{-1}=-\mu \chi^{\dagger}(t, \mathbf{r}) \hat{I} \chi(t, \mathbf{r}) .
$$

One can also check that the mass term with $\sigma_{2}$ also breaks $C$, whereas $\sigma_{1}$ mass term does not break $C$.

\section{Time-reversal symmetry}

The operation of time reversal for the electromagnetic field is defined as

$$
A_{0}(t, \mathbf{r}) \rightarrow \mathcal{T} A_{0}(t, \mathbf{r}) \mathcal{T}^{-1}=A_{0}(-t, \mathbf{r}), \quad A_{1,2}(t, \mathbf{r}) \rightarrow \mathcal{T} A_{1,2}(t, \mathbf{r}) \mathcal{T}^{-1}=-A_{1,2}(-t, \mathbf{r}) .
$$

We determine a matrix $T$ responsible for the time-reversal transformation of spinors

$$
\chi(t, \mathbf{r}) \rightarrow \mathcal{T} \chi(t, \mathbf{r}) \mathcal{T}^{-1}=T \chi(-t, \mathbf{r}), \quad \chi^{\dagger}(t, \mathbf{r}) \rightarrow \mathcal{T} \chi^{\dagger}(t, \mathbf{r}) \mathcal{T}^{-1}=\chi^{\dagger}(-t, \mathbf{r}) T
$$

demanding that the current components transform as follows

$$
\chi^{\dagger}(t, \mathbf{r}) \alpha_{\nu} \chi(t, \mathbf{r}) \rightarrow \chi^{T \dagger}(-t, \mathbf{r}) \alpha_{\nu}^{*} \chi^{T}(-t, \mathbf{r})=\chi^{\dagger}(-t, \mathbf{r}) T \alpha_{\nu}^{*} T \chi(-t, \mathbf{r})=\chi^{\dagger}(-t, \mathbf{r}) \tilde{\alpha}_{\nu} \chi(-t, \mathbf{r}),
$$

where

$$
\alpha_{\nu}=\left(\hat{I}, \sigma_{3}, \sigma_{2}\right), \quad \tilde{\alpha}_{\nu}=\left(\alpha_{0},-\alpha_{1},-\alpha_{2}\right) .
$$

The time-reversal operation (2.30) can be written down using $T=\sigma_{2}$ and one can see that the Lagrangian $\mathcal{L}$ is invariant with respect to this symmetry

$$
\mathcal{L}\left[\chi^{\dagger}(t, \mathbf{r}), \chi(t, \mathbf{r}), A_{\nu}(t, \mathbf{r})\right] \rightarrow \mathcal{L}\left[\chi^{T \dagger}(-t, \mathbf{r}), \chi^{T}(-t, \mathbf{r}), A_{\nu}^{T}(-t, \mathbf{r})\right]=\mathcal{L}\left[\chi^{\dagger}(t, \mathbf{r}), \chi(t, \mathbf{r}), A_{\nu}(t, \mathbf{r})\right],
$$

while the $\sigma_{1}$ and $\sigma_{2}$ mass terms would break it

$$
\chi^{\dagger}(t, \mathbf{r}) \sigma_{1,2} \chi(t, \mathbf{r}) \rightarrow \mathcal{T} \chi^{\dagger}(t, \mathbf{r}) \sigma_{1,2} \chi(t, \mathbf{r}) \mathcal{T}^{-1}=-\chi^{\dagger}(-t, \mathbf{r}) \sigma_{1,2} \chi(-t, \mathbf{r}) .
$$

One can check that if we use notations corresponding to those of Ref. [20], $P$ and $T$ operations introduced here agree with the transformations given in Ref. [20]. The time-reversal symmetry operation (2.30) for the continuum nodal Lagrangian should be identified with the combined symmetry operation (2.6) that leaves the Hamiltonian (2.5) invariant. We note that, in principle, the time-reversal transformation should also flip the spin $s \rightarrow-s$, but since all considered expressions assume summation over two spin components, we do not include this spin flip in the transformation of Dirac spinors.

Parity and time-reversal symmetries can still be broken by the mass (gap) term $\sim \sigma_{1}$. In particular, this mass term may be generated in an external magnetic field due to the so called magnetic catalysis phenomenon [21]. 


\section{Final form of the nodal Lagrangian}

We choose the vector potential for the external magnetic field $\mathbf{B}$ in the symmetric Poincaré gauge $\mathbf{A}=\left(-\frac{B}{2} x_{2}, \frac{B}{2} x_{1}\right)$, so that the field $\mathbf{B}$ is perpendicular to $\mathrm{CuO}_{2}$ planes. To consider also a possibility of magnetic catalysis [21] we add to the Lagrangian (2.24) the interaction term originating from particle-hole attraction

$$
\mathcal{L}_{\text {int }}=\frac{g}{2}\left(\chi^{\dagger}(x) \sigma_{1} \chi(x)\right)^{2}, \quad x=(t, \mathbf{r}) .
$$

The simplest way to treat the interaction (2.35) is to introduce Hubbard-Stratonovich field $\varphi(x)=-g \chi^{\dagger}(x) \sigma_{1} \chi(x)$, so that the Lagrangian (2.24) becomes

$$
\mathcal{L}=\chi^{\dagger}(x)\left[i \alpha_{\nu} D_{\nu}-\sigma_{1} \varphi(x)\right] \chi(x)-\frac{\varphi^{2}(x)}{2 g},
$$

where the covariant derivatives are

$$
D_{\nu}= \begin{cases}\hbar \partial_{t}-i e A_{0}(x), & \nu=0 \\ v_{F}\left(\hbar \partial_{x}-i \frac{e}{c} A_{1}(x)\right), & \nu=1 \\ v_{\Delta}\left(\hbar \partial_{y}-i \frac{e}{c} A_{2}(x)\right), & \nu=2\end{cases}
$$

and $\alpha$ matrices were defined in Eq. (2.32).

Finally, to simplify further calculations and to make a direct link with $\mathrm{QED}_{2+1}$ it is convenient to introduce Dirac conjugated spinor $\bar{\chi}(x)=\chi^{\dagger}(x) \sigma_{1}$ and rewrite the Lagrangian (2.36) as

$$
\mathcal{L}=\bar{\chi}(x)\left[i \gamma^{\nu} D_{\nu}-\varphi(x)\right] \chi(x)-\frac{\varphi^{2}(x)}{2 g},
$$

where the $\gamma$ matrices are

$$
\gamma^{\nu}=\left(\sigma_{1},-i \sigma_{2}, i \sigma_{3}\right), \quad\left\{\gamma^{\mu}, \gamma^{\nu}\right\}=2 \hat{I} g^{\mu \nu}, \quad g^{\mu \nu}=\operatorname{diag}(1,-1,-1) .
$$

Note that the Zeeman term, if necessary, can be added explicitly both to the original Hamiltonian (2.1) and the Lagrangian (2.38). Here, however, this term is neglected.

\section{GREEN'S FUNCTION IN AN EXTERNAL MAGNETIC FIELD}

To derive the transport coefficients we need an explicit representation for the fermionic Green's function in an external field. The calculation, following the Schwinger (proper time) approach [22], is sketched in Appendix \$ and the result is (we set for convenience $v_{F}=v_{D}=1$ and restore them when it is needed according to the prescription given in Appendix B

$$
S(x-y)=\exp \left(i e \int_{y}^{x} A_{\lambda}^{\mathrm{ext}} d z^{\lambda}\right) \tilde{S}(x-y),
$$

where the translation invariant part $\tilde{S}$ in the Matsubara frequency-momentum representation is given by

$$
\begin{aligned}
\tilde{S}(i \omega, \mathbf{p})= & -\int_{0}^{\infty} d s \exp \left[-s\left(\Delta^{2}-(i \omega)^{2}+\mathbf{p}^{2} \frac{\tanh (e B s)}{e B s}\right)\right] \\
& \times\left[\left(i \omega \gamma^{0}-p_{1} \gamma^{1}-p_{2} \gamma^{2}+\Delta+\frac{1}{i}\left(p_{2} \gamma^{1}-p_{1} \gamma^{2}\right) \tanh (e B s)\right)\left(1+\frac{1}{i} \gamma^{1} \gamma^{2} \tanh (e B s)\right)\right] .
\end{aligned}
$$

Here we replaced the Hubbard-Stratonovich field $\varphi(x)$ by its mean-field value $\Delta=\langle\varphi(x)\rangle$. The chemical potential $\mu$ has to be taken into account via the shift $i \omega \rightarrow i \omega+\mu$ [see Eq. (B3]) in Appendix [B].

As already mentioned, the gap $\Delta$ can be generated by an external magnetic field 21]. The value of the gap has to be determined from the minimum condition of the corresponding effective potential, see e.g. [21, 23, 24]. Here, 
however, we are mainly interested in the case of $\Delta=0$ and will concentrate on $\mu$ and $T$ dependences of the Lorenz ratio $L$.

The propagator (3.2) can be decomposed over the Landau level poles [21, 25] (see also Appendix A for the details):

$$
\tilde{S}(i \omega, \mathbf{p})=\exp \left(-\frac{\mathbf{p}^{2}}{|e B|}\right) \sum_{n=0}^{\infty}(-1)^{n} \frac{S_{n}(B, i \omega, \mathbf{p})}{(i \omega)^{2}-\Delta^{2}-2|e B| n}
$$

where

$$
S_{n}(B, i \omega, \mathbf{p})=2\left(\Delta+i \omega \gamma^{0}\right)\left[P_{-} L_{n}\left(2 \frac{\mathbf{p}^{2}}{|e B|}\right)-P_{+} L_{n-1}\left(2 \frac{\mathbf{p}^{2}}{|e B|}\right)\right]+4\left(p_{1} \gamma^{1}+p_{2} \gamma^{2}\right) L_{n-1}^{1}\left(2 \frac{\mathbf{p}^{2}}{|e B|}\right)
$$

with $P_{ \pm}=\left[1 \pm \operatorname{sgn}(e B) i \gamma^{1} \gamma^{2}\right] / 2=\left(1 \mp \sigma_{1}\right) / 2$ being projectors and $L_{n}, L_{n}^{1}$ Laguerre's polynomials $\left(L_{-1}^{1} \equiv 0\right)$. In what follows for convenience we take $e B>0$. The spectral function $A_{D}(\omega, \mathbf{p})$ associated with the translation-invariant part $\tilde{S}$ of the Green's function is defined as

$$
A_{D}(\omega, \mathbf{p})=\frac{1}{2 \pi i}\left[\tilde{S}^{A}(\omega-i 0, \mathbf{p})-\tilde{S}^{R}(\omega+i 0, \mathbf{p})\right]
$$

where the retarded, $\tilde{S}^{R}$, and advanced, $\tilde{S}^{A}$, Greens' functions are $\tilde{S}^{R}(\omega+i 0, \mathbf{p})=\tilde{S}(i \omega \rightarrow \omega+i 0, \mathbf{p})$ and $\tilde{S}^{A}(\omega-i 0, \mathbf{p})=$ $\tilde{S}(i \omega \rightarrow \omega-i 0, \mathbf{p})$. Using the definition (3.5) one obtains the spectral function associated with the "Dirac" fermion Green's function $\langle\chi \bar{\chi}\rangle$ (3.3) for a clean system

$$
A_{D}(\omega, \mathbf{p})=\exp \left(-\frac{\mathbf{p}^{2}}{e B}\right) \sum_{n=0}^{\infty}(-1)^{n}\left[\frac{\left(\gamma^{0} M_{n}+\Delta\right) f_{1}(\mathbf{p})+f_{2}(\mathbf{p})}{2 M_{n}} \delta\left(\omega-M_{n}\right)+\frac{\left(\gamma^{0} M_{n}-\Delta\right) f_{1}(\mathbf{p})-f_{2}(\mathbf{p})}{2 M_{n}} \delta\left(\omega+M_{n}\right)\right]
$$

where $M_{n}=\sqrt{\Delta^{2}+2 e B n}$ and

$$
f_{1}(\mathbf{p})=2\left[P_{-} L_{n}\left(2 \frac{\mathbf{p}^{2}}{e B}\right)-P_{+} L_{n-1}\left(2 \frac{\mathbf{p}^{2}}{e B}\right)\right], \quad f_{2}(\mathbf{p})=4\left(p_{1} \gamma^{1}+p_{2} \gamma^{2}\right) L_{n-1}^{1}\left(2 \frac{\mathbf{p}^{2}}{e B}\right) .
$$

To consider transport phenomena for a more realistic case one should introduce into the theory the effect of scattering on impurities. In general this can be done by considering dressed fermion propagators that include the selfenergy $\Sigma(\omega)$ due to the scattering on impurities. This self-energy in turn has to be found self-consistently by solving the corresponding Schwinger-Dyson equation. This scheme was in fact used in the Ref. 8], and the WF violation obtained by $\mathrm{KC}$ at finite temperatures in the unitary limit is essentially due to the nontrivial frequency dependence of the scattering rate $\Gamma(\omega)=-\operatorname{Im} \Sigma^{R}(\omega)$. As mentioned in Introduction, here we choose the case of constant width $\Gamma=\Gamma(\omega=0)=1 /(2 \tau), \tau$ being a mean free time of quasiparticles, so that the $\delta$-like quasiparticle peaks in Eq. (3.6) acquire a Lorentzian shape:

$$
\delta\left(\omega \pm M_{n}\right) \rightarrow \frac{\Gamma}{\pi} \frac{1}{\left(\omega \pm M_{n}\right)^{2}+\Gamma^{2}}
$$

Such a broadening of Landau levels was found to be a rather good approximation valid in not very strong magnetic fields [26].

Finally, we establish a link between the Green's function (3.1) considered here and the Green's function (2.20) used in the preceding section. Taking into account that the average $\left\langle\chi \chi^{\dagger}\right\rangle$ is related to the "Dirac's average" via $\left\langle\chi \chi^{\dagger}\right\rangle=\langle\chi \bar{\chi}\rangle \gamma^{0}$ one obtains for $B=0$ :

$$
G(i \omega, \mathbf{p})=\tilde{S}\left(i \omega_{m}, \mathbf{p} ; B=0\right) \gamma^{0}=\frac{i \omega+p_{1} \sigma_{3}+p_{2} \sigma_{2}+\Delta \sigma_{1}}{(i \omega)^{2}-\mathbf{p}^{2}-\Delta^{2}},
$$

so that restoring $v_{F}, v_{D}$, replacing $i \omega_{n} \rightarrow i \omega_{n}+\mu$, and setting $\Delta=0$ we recover the Green's function (2.21). 


\section{ELECTRICAL CONDUCTIVITY}

\section{A. General expression for electrical conductivity}

The frequency dependent longitudinal electrical conductivity can be calculated from the current-current correlation function

$$
\Pi_{\alpha \beta}^{C C}(i \Omega)=-\int_{0}^{\beta} d \tau e^{i \Omega \tau}\left\langle T_{\tau} j_{\alpha}^{\dagger}(\tau, \mathbf{0}) j_{\beta}(0, \mathbf{0})\right\rangle
$$

by means of the Kubo formula [27]

$$
\sigma(\Omega)=-\frac{\operatorname{Im} \Pi_{R}^{C C}(\Omega+i 0)}{\Omega},
$$

where $\Pi_{R}^{C C}(\Omega+i 0)=\Pi^{C C}(i \Omega \rightarrow \Omega+i 0)$ is the longitudinal polarization [see Eq. (B14)].

The expression for the electrical current operator for the Hamiltonian (2.1) was already derived in Eq. (2.14). Having the current, we are in a position to calculate the current-current correlation function (4.1), which is given by the bubble diagram. The influence of the impurity vertex corrections on the transport properties of another $(d \mathrm{SC})$ nodal system was considered in Ref. [17]. The calculation of the bare bubble polarization function is in fact similar for all transport coefficients in $d \mathrm{SC}$ and DDW nodal liquids, so that it is convenient to define a generalized polarization function $\Pi^{g g^{\prime}}(\Omega)$ that depends on the coupling parameters $g, g^{\prime}$ and the generalized velocity $\mathbf{V}(\mathbf{k})$. The polarization function is calculated in Appendix [B] Using the general result (B11) for the case of interest, $g=g^{\prime}=e$ in the limit $\Omega \rightarrow 0$, we find that the dc conductivity of isotropic system is given by

$$
\sigma=\pi e^{2} \int_{\mathrm{RBZ}} \frac{d^{2} k}{(2 \pi)^{2}} \int_{-\infty}^{\infty} d \omega\left(-n_{F}^{\prime}(\omega-\mu)\right) \operatorname{tr}\left[A(\omega, \mathbf{k}) V_{\alpha}(\mathbf{k}) A(\omega, \mathbf{k}) V_{\alpha}(\mathbf{k})\right],
$$

where $-n_{F}^{\prime}(\omega-\mu)=(1 / 4 T) \cosh ^{-2}[(\omega-\mu) / 2 T]$ is the derivative of the Fermi distribution and summation over the dummy variable is implied.

At this point it is instructive to compare Eq. 4.3) with the expression for the conductivity obtained by Langer [Eq. (4.8) of Ref. 28]] in the bubble approximation. Langer's expression has the same form as Eq. (4.3) except the matrix-valued $e V_{\alpha}(\mathbf{k})$ is replaced by the vertex with coinciding fermion momenta and energies

$$
\Lambda_{i}(\mathbf{k}, \omega)=e \frac{k_{i}}{m}-e \frac{\partial}{\partial k_{i}} \Sigma(\mathbf{k}, \omega)
$$

where $\Sigma(\mathbf{k}, \omega)$ is the usual self-energy [he considered the quadratic dispersion law, so that $\partial \epsilon(\mathbf{k}) / \partial k_{i}=k_{i} / m$ ]. In fact Eq. (4.4) is a direct consequence of the Ward identity. Thus knowing the self-energy one immediately obtains the dc conductivity in the bubble approximation. Furthermore, the bubble conductivity of Ref. [28] becomes exact in the zero-temperature limit.

Since in the mean-field approximation $\Sigma(\mathbf{k}, \omega)$ is replaced by the DDW gap $\sigma_{2} D(\mathbf{k})$, Langer's expression reduces to Eq. (4.3). It is also evident from Eq. (4.4) that for the momentum-independent self-energy the bubble conductivity coincides with the conductivity calculated in the bare bubble approximation, but clearly this is not the case of the DDW gap. Expression (4.3) can also be derived in the lowest order approximation from a general expression for the dc conductivity, with the vertex corrections taken into account, obtained by Eliashberg [29].

Making the nodal approximation (see Eq. (B16) ) we get

$$
\begin{aligned}
\sigma(B, T) & =\frac{2 \pi e^{2}}{v_{F} v_{D}} \int \frac{d^{2} p}{(2 \pi)^{2}} \int_{-\infty}^{\infty} d \omega\left(-n_{F}^{\prime}(\omega-\mu)\right) \\
& \times\left(v_{F}^{2} \operatorname{tr}\left[A_{D}(\omega, \mathbf{p}) \gamma^{1} A_{D}(\omega, \mathbf{p}) \gamma^{1}\right]+v_{D}^{2} \operatorname{tr}\left[A_{D}(\omega, \mathbf{p}) \gamma^{2} A_{D}(\omega, \mathbf{p}) \gamma^{2}\right]\right) .
\end{aligned}
$$

Finally we note that one could also calculate conductivity directly working with the nodal Lagrangian (2.38) and expressing the bubble in terms of the corresponding electrical current operator

$$
j_{x}=e v_{F} \bar{\chi}_{s} \gamma^{1} \chi_{s}, \quad j_{y}=e v_{D} \bar{\chi}_{s} \gamma^{2} \chi_{s}
$$

which is also valid in the presence of an external field. This way of calculation is exactly the same as in Ref. [] and final result agrees with (4.5). 


\section{B. Calculation of conductivity}

Straightforward calculation of the trace in Eq. (4.5), with $A_{D}(\omega, \mathbf{k})$ from Eqs. (3.6) and (3.7) but with the $\delta$ functions in Eq. (3.6) replaced by the Lorentzians (3.8), gives

$$
\begin{aligned}
& \operatorname{tr}\left[A_{D}(\omega, \mathbf{p}) \gamma^{1,2} A_{D}(\omega, \mathbf{p}) \gamma^{1,2}\right]=\frac{4 \Gamma^{2}}{\pi^{2}} \exp \left(-\frac{2 \mathbf{p}^{2}}{e B}\right) \\
& \times \sum_{n, m=0}^{\infty}(-1)^{n+m+1} \frac{\left[\left(\omega^{2}+M_{n}^{2}+\Gamma^{2}\right)\left(\omega^{2}+M_{m}^{2}+\Gamma^{2}\right)-4 \omega^{2} \Delta^{2}\right]\left[L_{n} L_{m-1}+L_{n-1} L_{m}\right] \mp 32\left(p_{1}^{2}-p_{2}^{2}\right) L_{n-1}^{1} L_{m-1}^{1}}{\left[\left(\omega^{2}+M_{n}^{2}+\Gamma^{2}\right)^{2}-4 \omega^{2} M_{n}^{2}\right]\left[\left(\omega^{2}+M_{m}^{2}+\Gamma^{2}\right)^{2}-4 \omega^{2} M_{m}^{2}\right]}
\end{aligned}
$$

where all Laguerre's polynomials depend on $\frac{2 \mathbf{p}^{2}}{e B}$, and the minus sign corresponds to $\gamma^{1}$ and the plus sign to $\gamma^{2}$, respectively.

The integration over momentum $p$ can be easily done after extending the upper limit of integration to $\infty$, so that one can use the orthogonality of Laguerre's polynomials

$$
\int_{0}^{\infty} d x e^{-x} x^{\alpha} L_{m}^{\alpha}(x) L_{n}^{\alpha}(x)=\Gamma(1+\alpha) \frac{(n+\alpha) !}{n ! \alpha !} \delta_{m n} \quad \text { with } \quad x=\frac{2 \mathbf{p}^{2}}{e B}
$$

and obtain electrical conductivity in terms of the sum over the transitions between neighboring Landau levels

$$
\begin{aligned}
\sigma(B, T) & =e^{2} \frac{v_{F}^{2}+v_{D}^{2}}{v_{F} v_{D}} \frac{e B \Gamma^{2}}{2 \pi^{2} T} \\
& \times \sum_{n=0}^{\infty} \int_{-\infty}^{\infty} d \omega \frac{1}{\cosh ^{2} \frac{\omega-\mu}{2 T}} \frac{\left(\omega^{2}+M_{n}^{2}+\Gamma^{2}\right)\left(\omega^{2}+M_{n+1}^{2}+\Gamma^{2}\right)-4 \omega^{2} \Delta^{2}}{\left[\left(\omega^{2}+M_{n}^{2}+\Gamma^{2}\right)^{2}-4 \omega^{2} M_{n}^{2}\right]\left[\left(\omega^{2}+M_{n+1}^{2}+\Gamma^{2}\right)^{2}-4 \omega^{2} M_{n+1}^{2}\right]}
\end{aligned}
$$

The sum over $n$ in Eq. (4.9) can be expressed via the digamma function $\psi$ as described in Ref. 23], and the final expression for the electrical conductivity is

$$
\sigma=e^{2} \alpha \int_{-\infty}^{\infty} \frac{d \omega}{4 T \cosh ^{2} \frac{\omega-\mu}{2 T}} \mathcal{A}(\omega, B, \Gamma, \Delta(B))
$$

where we introduced the function $\mathcal{A}$

$$
\begin{aligned}
\mathcal{A}(\omega, B, \Gamma, \Delta(B))=\frac{1}{\pi^{2}} \frac{\Gamma^{2}}{(e B)^{2}+(2 \omega \Gamma)^{2}} & \left\{2 \omega^{2}+\frac{\left(\omega^{2}+\Delta^{2}+\Gamma^{2}\right)(e B)^{2}-2 \omega^{2}\left(\omega^{2}-\Delta^{2}+\Gamma^{2}\right) e B}{\left(\omega^{2}-\Delta^{2}-\Gamma^{2}\right)^{2}+4 \omega^{2} \Gamma^{2}}\right. \\
& \left.-\frac{\omega\left(\omega^{2}-\Delta^{2}+\Gamma^{2}\right)}{\Gamma} \operatorname{Im} \psi\left(\frac{\Delta^{2}+\Gamma^{2}-\omega^{2}-2 i \omega \Gamma}{2 e B}\right)\right\}
\end{aligned}
$$

and

$$
\alpha=\frac{v_{F}}{v_{D}}+\frac{v_{D}}{v_{F}} .
$$

Another representation of Eq. (4.11) can be obtained using the series representation of $\psi$ function and writing the expression in curly brackets in fractions of $1 /\left(\Gamma^{2}+x^{2}\right)$,

$$
\begin{aligned}
\mathcal{A}(\omega, B, \Gamma, \Delta(B))=\frac{1}{\pi^{2}} \frac{\Gamma^{2}}{(e B)^{2}+(2 \omega \Gamma)^{2}} & \left\{2 \omega^{2}+\frac{\frac{(e B)^{2}}{2}+e B \omega(\omega+\Delta)}{(\omega+\Delta)^{2}+\Gamma^{2}}+\frac{\frac{(e B)^{2}}{2}+e B \omega(\omega-\Delta)}{(\omega-\Delta)^{2}+\Gamma^{2}}\right. \\
& \left.+e B \omega \sum_{n=1}^{\infty} \frac{1}{M_{n}}\left[\frac{\Delta^{2}+M_{n}^{2}+2 \omega M_{n}}{\left(\omega+M_{n}\right)^{2}+\Gamma^{2}}+\frac{2 \omega M_{n}-\Delta^{2}-M_{n}^{2}}{\left(\omega-M_{n}\right)^{2}+\Gamma^{2}} .\right]\right\}
\end{aligned}
$$

The representation (4.13) is particularly convenient for studying the narrow width limit $\Gamma \ll T$, $\sqrt{e B}$ when we can replace the fractions $\Gamma /\left(\Gamma^{2}+x^{2}\right)$ by $\pi \delta(x)$ :

$$
\begin{aligned}
\mathcal{A}(\omega, B, \Gamma, \Delta(B))=\frac{\Gamma}{\pi} & \left\{\frac{1}{(e B)^{2}+4 \Delta^{2} \Gamma^{2}}\left[\frac{(e B)^{2}}{2} \delta(\omega+\Delta)+\frac{(e B)^{2}}{2} \delta(\omega-\Delta)\right]\right. \\
& \left.+\sum_{n=1}^{\infty} \frac{2(e B)^{2} n}{(e B)^{2}+4\left(\Delta^{2}+2 e B n\right) \Gamma^{2}}\left[\delta\left(\omega+M_{n}\right)+\delta\left(\omega-M_{n}\right)\right]\right\},
\end{aligned}
$$


where we kept $\Gamma^{2}$ in the denominators in order to be able to reproduce a smooth behavior of $\sigma(B)$ and $\kappa(B)$ in the limit $B \rightarrow 0$. We are now in a position to study different asymptotic regimes defined by different relations among the parameters $\Gamma, T, \mu, B$, and $\Delta$.

\section{Zero magnetic field}

We begin with the limit of vanishing magnetic field $(B=0)$. Using the large $z$ asymptote of the $\psi$ function

$$
\psi(z)=\ln z-\frac{1}{2 z}-\frac{1}{12 z^{2}}+\frac{1}{120 z^{4}}+O\left(\frac{1}{z^{6}}\right)
$$

we obtain

$$
\mathcal{A}(\omega, B=0, \Gamma, \Delta)=\frac{1}{2 \pi^{2}}\left[1+\frac{\omega^{2}-\Delta^{2}+\Gamma^{2}}{2|\omega| \Gamma}\left(\frac{\pi}{2}-\arctan \frac{\Delta^{2}+\Gamma^{2}-\omega^{2}}{2|\omega| \Gamma}\right)\right] .
$$

Putting $\Delta=0$ also in Eq. (4.16), we get

$$
\begin{gathered}
\mathcal{A}(\omega, B=0, \Gamma, \Delta=0)=\frac{1}{2 \pi^{2}}\left[1+\frac{\omega^{2}+\Gamma^{2}}{\omega \Gamma} \arctan \frac{\omega}{\Gamma}\right] . \\
\text { 1. } \quad \text { Limit } T \rightarrow 0
\end{gathered}
$$

The limit $T \rightarrow 0$ is significantly simplified by the fact that $\cosh ^{-2}$ term in Eq. (4.10) can be replaced by the $\delta$ function

$$
\sigma=e^{2} \alpha \mathcal{A}(\mu, B=0, \Gamma, \Delta)
$$

where $\mathcal{A}$ is given by Eq. (4.16). For the case of zero gap $\Delta=0$ using Eq. (4.17) we obtain that for $\mu=0$

$$
\sigma(\mu=0)=\frac{e^{2} \alpha}{\pi^{2}}
$$

and for $|\mu| \gg \Gamma$

$$
\sigma=\frac{e^{2} \alpha}{4 \pi} \frac{|\mu|}{\Gamma}
$$

Comparing Eq. (4.19) with Eq. (57) of YN, one can see that our result is twice as large, but contains the same prefactor $\alpha$ that reflects the fact that the electrical current operator has the component $\sim \mathbf{v}_{D}$. On the other hand, the result of $\mathrm{KC}$ is $\sim v_{F} / v_{D}$ due to the fact that they took the current without the $\mathbf{v}_{D}$ component, and it is twice as large as Eq. (4.19) because KC integrated over the full Brillouin zone instead of the reduced one. Expression (4.19) can also be compared with its superconducting counterpart [17] $\sigma_{S C}=\left(e^{2} / \pi^{2}\right) v_{F} / v_{\Delta}$, where $v_{\Delta}$ is the $d$ SC gap velocity. As one can see the numerical prefactor is exactly the same, but there is no $v_{\Delta} / v_{F}$ term in $\sigma_{S C}$ because the electrical current in the $d \mathrm{SC}$ state is $\sim \mathbf{v}_{F}$.

Finally one can also compare our $\mu \neq 0$ expression (4.20) with Eq. (13) of KC:

$$
\sigma\left(T \ll D_{0}\right) \simeq \frac{e^{2}}{2 \pi} \frac{v_{F}}{v_{D}} \frac{D_{0}}{\gamma_{0}},
$$

where in the Born limit $\gamma_{0}$ is related to $\Gamma(\omega)$ via $\Gamma(\omega)=\gamma_{0}\left[(\omega+\mu) / D_{0}\right]$. Substituting the value of $\Gamma(0)$ in Eq. (4.20) one can see that it reduces to Eq. (4.21) except for the abovementioned differences in the factors. 


\section{Limit $T \ll \Gamma$}

In order to obtain low-temperature corrections to conductivities it is convenient to apply the Sommerfeld expansion

$$
\begin{aligned}
& \int_{-\infty}^{\infty} \frac{(\omega-\mu)^{n}}{4 T \cosh ^{2} \frac{\omega-\mu}{2 T}} f(\omega) d \omega \simeq \int_{-\infty}^{\infty} \frac{(\omega-\mu)^{n}}{4 T \cosh ^{2} \frac{\omega-\mu}{2 T}} d \omega f(\mu) \\
& +\int_{-\infty}^{\infty} \frac{(\omega-\mu)^{n+1}}{4 T \cosh ^{2} \frac{\omega-\mu}{2 T}} d \omega f^{\prime}(\mu)+\frac{1}{2} \int_{-\infty}^{\infty} \frac{(\omega-\mu)^{n+2}}{4 T \cosh ^{2} \frac{\omega-\mu}{2 T}} d \omega f^{\prime \prime}(\mu)+O\left(\left(\frac{T}{\max (\mu, \Gamma)}\right)^{n+3}\right) .
\end{aligned}
$$

Here the function $f$ should be nonsingular and not too rapidly varying in the vicinity of $\omega=\mu$ and the integrals on the right hand side of Eq. (4.22) are

$$
\int_{-\infty}^{\infty} \frac{d \omega}{4 T \cosh ^{2} \frac{\omega-\mu}{2 T}}=1, \quad \int_{-\infty}^{\infty} \frac{d \omega(\omega-\mu)^{2}}{4 T \cosh ^{2} \frac{\omega-\mu}{2 T}}=\frac{\pi^{2} T^{2}}{3}, \quad \int_{-\infty}^{\infty} \frac{d \omega(\omega-\mu)^{4}}{4 T \cosh ^{2} \frac{\omega-\mu}{2 T}}=\frac{7 \pi^{4} T^{4}}{15}
$$

It is easy to derive the next to the leading term in $T^{2}$ to the conductivity (4.18) for $\Delta=0$,

$$
\sigma=e^{2} \alpha\left[\mathcal{A}(\omega=\mu, 0, \Gamma, 0)+\frac{\pi^{2} T^{2}}{6} \mathcal{A}_{\omega}^{\prime \prime}(\omega=\mu, 0, \Gamma, 0)\right],
$$

where the derivatives of $\mathcal{A}$ with respect to $\omega$ are

$$
\begin{aligned}
& \mathcal{A}_{\omega}^{\prime}(\omega, 0, \Gamma, 0)=\frac{1}{2 \pi^{2} \omega}\left(1+\frac{\omega^{2}-\Gamma^{2}}{\omega \Gamma} \arctan \frac{\omega}{\Gamma}\right) \\
& \mathcal{A}_{\omega}^{\prime \prime}(\omega, 0, \Gamma, 0)=\frac{1}{\pi^{2} \omega^{2}}\left(\frac{\Gamma}{\omega} \arctan \frac{\omega}{\Gamma}-\frac{\Gamma^{2}}{\omega^{2}+\Gamma^{2}}\right) .
\end{aligned}
$$

For $\mu=0$ case Eq. (4.24) gives the $T^{2}$ correction to the expression (4.19):

$$
\sigma(\mu=0)=\frac{e^{2} \alpha}{\pi^{2}}\left[1+\frac{\pi^{2}}{9} \frac{T^{2}}{\Gamma^{2}}\right], \quad T \ll \Gamma .
$$

\section{3. $\quad$ Limit $T \gg \Gamma$}

For $\Gamma \rightarrow 0$ from Eq. (4.16) we get

$$
\mathcal{A}(\omega, B=0, \Gamma, \Delta)=\frac{1}{4 \pi^{2}} \frac{\omega^{2}-\Delta^{2}}{|\omega| \Gamma} \pi \theta\left(\omega^{2}-\Delta^{2}\right) .
$$

For the $\Delta=0$ case retaining more terms in the expansion of Eq. (4.17), we obtain

$$
\mathcal{A}(\omega, B=0, \Gamma, \Delta=0)=\frac{1}{4 \pi}\left(\frac{|\omega|}{\Gamma}+\frac{\Gamma}{|\omega|}\right) .
$$

Substituting Eq. (4.28) in the expression (4.10) for $\sigma$ we arrive at

$$
\sigma=e^{2} \alpha \frac{T}{2 \pi \Gamma} \ln \left(2 \cosh \frac{\mu}{2 T}\right), \quad T \gg \Gamma .
$$

As one can easily see for $\mu \gg T$ Eq. (4.29) reduces to Eq. (4.20) reflecting the fact that when $\mu$ is the largest parameter the value of conductivity is not sensitive to the relation between $T$ and $\Gamma$.

As mentioned before, the magnetic catalysis phenomenon [21, 23, 24] implies that the gap $\Delta$ is generated only in the presence of an external field. Nevertheless, one can also study the consequences of the gap opening even for $B=0$ 
case to gain a deeper insight to a more complicated case $B, \Delta \neq 0$. In particular, considering the $\Delta \gg T$ case, we obtain from Eqs. (4.27) and (4.10) that

$$
\sigma=\frac{e^{2} \alpha}{16 \pi T \Gamma} \int_{\Delta}^{\infty} d \omega \frac{\omega^{2}-\Delta^{2}}{\omega}\left[\frac{1}{\cosh ^{2} \frac{\omega-\mu}{2 T}}+(\mu \rightarrow-\mu)\right] \simeq \frac{e^{2} \alpha}{4 \pi \Gamma}\left\{\begin{array}{cc}
\frac{\mu^{2}-\Delta^{2}}{|\mu|}-\frac{\pi^{2} T^{2} \Delta^{2}}{3 \mu^{2}|\mu|}, & |\mu|>\Delta \\
2 T \ln 2, & |\mu|=\Delta \\
4 T \cosh \frac{\mu}{T} \exp \left(-\frac{\Delta}{T}\right), & |\mu|<\Delta .
\end{array}\right.
$$

It is clear from Eq. 4.30) that the opening of the gap $\Delta$ results in the thermally activated behavior of conductivity only for $|\mu|<\Delta$. As discussed in Sec. IVD3 this observation remains valid even in the presence of an external field.

\section{Nonzero magnetic field}

There are not so many cases available for analytical investigation for $B \neq 0$ and we have to integrate numerically Eq. (4.10) with $\mathcal{A}$ given by Eq. (4.11). Nevertheless in a few cases analytical expressions for the conductivity can be obtained and here we begin with considering these results.

\section{Limit $T \rightarrow 0$}

As one can notice, Eq. (4.18) is in fact valid even for nonzero $B$ because only the first of term of the Sommerfeld expansion (4.22) contributes, i.e.,

$$
\sigma=e^{2} \alpha \mathcal{A}(\mu, B, \Gamma, \Delta), \quad \forall \mu, \quad \Gamma \neq 0
$$

\section{Narrow width case}

To study the narrow width limit $\Gamma \rightarrow 0$ we use the representation (4.14) to arrive at

$$
\begin{aligned}
\sigma=e^{2} \alpha \frac{\Gamma}{4 \pi T} & \left\{\frac{(e B)^{2}}{2\left[(e B)^{2}+4 \Delta^{2} \Gamma^{2}\right]}\left[\frac{1}{\cosh ^{2} \frac{\Delta+\mu}{2 T}}+\frac{1}{\cosh ^{2} \frac{\Delta-\mu}{2 T}}\right]\right. \\
& \left.+\sum_{n=1}^{\infty} \frac{2(e B)^{2} n}{(e B)^{2}+4\left(\Delta^{2}+2 e B n\right) \Gamma^{2}}\left[\frac{1}{\cosh ^{2} \frac{\sqrt{\Delta^{2}+2 e B n}+\mu}{2 T}}+\frac{1}{\cosh ^{2} \frac{\sqrt{\Delta^{2}+2 e B n}-\mu}{2 T}}\right]\right\} .
\end{aligned}
$$

The fact that the conductivity is proportional to the scattering rate $\Gamma$ in the limit $\Gamma \rightarrow 0$ means that in contrast to the zero-field case (see Sec. IVC) it results from transitions of quasiparticles between neighboring cyclotron orbits.

Using Eq. (4.32) one easily gets the asymptotic expression for conductivity in the strong-field limit, $\sqrt{e B} \gtrsim 4 T$ for $\mu=\Delta=0$

$$
\sigma=e^{2} \alpha \frac{\Gamma}{4 \pi T}
$$

that shows that $\sigma$ becomes field independent in the strong field.

\section{Numerical calculation of electrical conductivity}

To investigate numerically the behavior of electrical and thermal conductivities, and to make comparison with experiment, we need to restore all model parameters, such as $\hbar, c, k_{B}, v_{F}$, and $v_{D}$ in Eqs. (4.10) and (4.11). As discussed after Eq. (B12) the prefactor $\alpha$ in Eq. (4.10) is already fixed and one should only substitute $T \rightarrow k_{B} T$, $\Gamma \rightarrow \hbar \Gamma$, and $e B \rightarrow\left(\hbar v_{F} v_{D} / c\right) e B$. It is convenient to measure all energetic quantities in K, which results in the following replacement:

$$
\sqrt{2 e B} \rightarrow \sqrt{\frac{\hbar v_{F} v_{D} 2 e B}{c}}[\mathrm{~K}]=63.9 \sqrt{\frac{v_{D}}{v_{F}}} v_{F}[\mathrm{eV} \cdot \AA] \sqrt{B[\text { Tesla }]}=4.206 \times 10^{-6} \sqrt{\frac{v_{D}}{v_{F}}} v_{F}[\mathrm{~cm} / \mathrm{s}] \sqrt{B[\text { Tesla }]}
$$


where in the first equality $v_{F}$ is given in $\mathrm{eV} \cdot \AA$ and in $\mathrm{cm} / \mathrm{s}$ in the second one. In particular, for $v_{F}=1.5 \mathrm{eV} \cdot \AA$ [this roughly agrees with the value of $t$ given after Eq. [2.21)] and $v_{F} / v_{D}=24$ using Eq. (4.34) we obtain that $e B \rightarrow 200 \cdot \mathrm{K}^{2} \cdot B$ [Tesla]. In what follows we use this estimate to compute all numerical expressions. There is a larger uncertainty for the value of $\Gamma$ that we could choose for our computations. For example, for clean YBCO monocrystals the estimated [30] value of the scattering rate due to impurities $\Gamma_{0} \sim 1-2 \mathrm{~K}$, so that we use the value $\Gamma=2 \mathrm{~K}$.

In Figs. 3 and 4 we show the temperature dependence of the conductivity for three different values of the applied field at half-filling (Fig. 31) and slightly away from it (Fig. (4). For $\mu=B=0$ the conductivity increases as the temperature $T$ grows. However, when the magnetic field is nonzero, the dependence $\sigma(T)$ becomes non-monotonic and there is first a decrease in $\sigma(T)$ as the temperature increases. For $\mu, B \neq 0$ (see Fig. 4 ) the conductivity slowly increases as the temperature grows and this dependence becomes almost flat as the field increases. This tendency agrees with Eqs. (4.32) and (4.33). In zero field both figures reveal an "insulator" (i.e., increasing with temperature) behavior in agreement with our analytical result (4.29). This behavior is a consequence of using a constant value for the scattering rate $\Gamma$ in our model. The growth of the conductivity with increasing temperature is directly related to the increasing number of thermally excited quasiparticles. The choice of temperature-independent $\Gamma$ might be reasonable in the narrow low-temperature region. It is essential, however, that the observed decrease and flattening $\sigma(T, B)$ in the nonzero external field are due to the assumption $\Gamma(B)=$ const discussed after Eq. (3.8) and are not related to the $\Gamma(T)=$ const approximation just mentioned.

Comparing Figs. 3 and 4 one can see that in zero field the increase of $|\mu|$ (opening of the pockets on the Fermi surface) leads to the increase of the conductivity. On the contrary, in the presence of the external field, the conductivity decreases as the pockets on the Fermi surface open. One can notice that the value of conductivity $\sigma$ at $\mu=T=0$ is field independent. This is due to the fact that for $\omega=0$, the function $\mathcal{A}$ [see Eq. (4.11) and Fig. 14] becomes field independent.

Using Eqs. (4.10) and (4.11) it is also possible to investigate the case of $\Delta \neq 0$. The main results for $B \neq 0$ can be foreseen from Eq. (4.30) and are the following. When $T,|\mu| \lesssim \Delta$ the behavior of $\sigma$ becomes thermally activated, i.e., governed by the factor $\sim \exp (-\Delta / T)$. In contrast, for $\Delta \lesssim|\mu|$ the behavior of $\sigma(T)$ is rather similar to the case of $\Delta=0$. This reflects the fact that the gap $\Delta$ induced by the magnetic catalysis, in contrast to a superconducting gap, is not tied to the Fermi surface and there are gapless excitations for $|\mu| \gtrsim \Delta$.

In Figs. $[5$ and $[6$ we show, respectively, the dependence of $\sigma(B)$ for three different values of $T$ and fixed $\mu$ and for three different values of $\mu$ at fixed $T$. Both figures show that $\sigma$ decreases as a function of $B$. This decrease reflects the fact mentioned before Eq. (4.9) that in the presence of magnetic field only transitions between neighboring Landau levels contribute into electrical (and thermal) conductivity. A further increase of the field and entering in the strong field regime, $\sqrt{e B} \gtrsim 4 T$ suppresses the transition between Landau levels, so that as one can see from Fig. [5] the conductivity $\sigma(B)$ becomes field independent. Furthermore for $T=10 \mathrm{~K}$ the value of normalized conductivity is $\approx 0.15$, in agreement with Eq. (4.33), which is derived under the assumption $\Gamma \ll T$ and taking into account only the transition between the lowest levels. In Fig. 6 we observe Shubnikov-de Haas oscillations of the conductivity that are due to the Landau-level crossing of the Fermi pockets (an analytical treatment of the magnetic oscillations in the conductivities will be presented elsewhere).

\section{THERMAL CONDUCTIVITY}

\section{A. General expression for thermal conductivity}

Thermal conductivity can be calculated from the the energy current-current correlation function

$$
\Pi_{\alpha \beta}^{E E}(i \Omega)=-\int_{0}^{\beta} d \tau e^{i \Omega \tau}\left\langle T_{\tau} j_{\alpha}^{E \dagger}(\tau, \mathbf{0}) j_{\beta}^{E}(0, \mathbf{0})\right\rangle,
$$

and the correlation function of energy current with the electrical current

$$
\Pi_{\alpha \beta}^{E C}(i \Omega)=-\int_{0}^{\beta} d \tau e^{i \Omega \tau}\left\langle T_{\tau} j_{\alpha}^{E \dagger}(\tau, \mathbf{0}) j_{\beta}(0, \mathbf{0})\right\rangle
$$

using a thermal Kubo formula 27, 31

$$
\frac{\kappa(\Omega)}{T}=-\frac{1}{T^{2}} \frac{\operatorname{Im} \Pi_{R}^{E E}(\Omega+i 0)}{\Omega}-S^{2}(\Omega) \sigma(\Omega, B, T)=-\frac{1}{T^{2}} \frac{\operatorname{Im} \prod_{R}^{E E}(\Omega+i 0)}{\Omega}-\frac{1}{T^{2}} \frac{\left[\operatorname{Im} \Pi_{R}^{E C}(\Omega+i 0)\right]^{2}}{\sigma(\Omega) \Omega^{2}} .
$$


Here $\Pi_{R}^{E E}(\Omega+i 0)=\Pi^{E E}(i \Omega \rightarrow \Omega+i 0)$ is the longitudinal polarization [see Eq. (B14)] and $S(\Omega, B, T)$ is the thermopower

$$
S(\Omega)=-\frac{1}{T} \frac{\operatorname{Im} \Pi_{R}^{E C}(\Omega)}{\operatorname{Im} \Pi_{R}^{C C}(\Omega)}
$$

with the longitudinal $\Pi_{R}^{E C}(\Omega+i 0)=\Pi^{E C}(i \Omega \rightarrow \Omega+i 0)$. The term with the thermal power $S$ ensures that the energy current is evaluated under the condition of vanishing electrical current [3] (see also Ref. 27]). Usually, for $T \ll \mu$ this term is considered to be unimportant because it is $\sim T^{2} / \mu^{2}$ times less than the first term of Eq. (5.3). It is also zero for $\mu=0$, but nevertheless its contribution is important for the case of interest, $|\mu| \lesssim T$.

The structure of the energy current (2.18) is similar to that of the electrical current (2.14). Hence, the calculation of the corresponding polarization functions (5.1) and (5.2) is almost identical to calculating the bubble (4.1). Using the general result (B16) to the case of interest: $g=g^{\prime}=\omega+\Omega / 2-\mu$, we arrive at

$$
\begin{aligned}
-\frac{\operatorname{Im} \Pi_{R}^{E E}(\Omega)}{T^{2} \Omega} & =\frac{\pi}{v_{F} v_{D}} \int \frac{d^{2} p}{(2 \pi)^{2}} \int_{-\infty}^{\infty} d \omega \frac{\tanh \frac{\omega-\mu+\Omega}{2 T}-\tanh \frac{\omega-\mu}{2 T}}{\Omega}\left(\frac{\omega-\mu+\Omega / 2}{T}\right)^{2} \\
& \times\left[v_{F}^{2} \operatorname{tr}\left[A_{D}(\omega, \mathbf{p}) \gamma^{1} A_{D}(\omega+\Omega, \mathbf{p}) \gamma^{1}\right]+v_{D}^{2} \operatorname{tr}\left[A_{D}(\omega, \mathbf{p}) \gamma^{2} A_{D}(\omega+\Omega, \mathbf{p}) \gamma^{2}\right]\right],
\end{aligned}
$$

and for $g=\omega+\Omega / 2-\mu, g^{\prime}=e$

$$
\begin{aligned}
-\frac{\operatorname{Im} \Pi_{R}^{E C}(\Omega)}{T \Omega} & =\frac{e \pi}{v_{F} v_{D}} \int \frac{d^{2} p}{(2 \pi)^{2}} \int_{-\infty}^{\infty} d \omega \frac{\tanh \frac{\omega-\mu+\Omega}{2 T}-\tanh \frac{\omega-\mu}{2 T}}{\Omega} \frac{\omega-\mu+\Omega / 2}{T} \\
& \times\left[v_{F}^{2} \operatorname{tr}\left[A_{D}(\omega, \mathbf{p}) \gamma^{1} A_{D}(\omega+\Omega, \mathbf{p}) \gamma^{1}\right]+v_{D}^{2} \operatorname{tr}\left[A_{D}(\omega, \mathbf{p}) \gamma^{2} A_{D}(\omega+\Omega, \mathbf{p}) \gamma^{2}\right]\right] .
\end{aligned}
$$

Similarly to the case of the electrical current, one could also obtain the energy current operator from the nodal Lagrangian (2.38) in the external magnetic field as done in Ref. [23],

$$
j_{x}^{E}=\frac{i v_{F}}{2}\left(\bar{\chi}_{s} \gamma^{1} \partial_{t} \chi_{s}-\partial_{t} \bar{\chi}_{s} \gamma^{1} \chi_{s}\right), \quad j_{y}^{E}=\frac{i v_{D}}{2}\left(\bar{\chi}_{s} \gamma^{2} \partial_{t} \chi_{s}-\partial_{t} \bar{\chi}_{s} \gamma^{2} \chi_{s}\right)
$$

to derive the expression (5.6). The current (5.7) corresponds to the current used by YN.

The calculation of thermal conductivity from Eq. (5.6) follows exactly the same route as for the electrical conductivity in Sec. IVB and finally we arrive at

$$
\begin{aligned}
\frac{\kappa(B, T)}{T}=\alpha & \left\{\int_{-\infty}^{\infty} d \omega\left(\frac{\omega-\mu}{T}\right)^{2} \frac{1}{4 T \cosh ^{2} \frac{\omega-\mu}{2 T}} \mathcal{A}(\omega, B, \Gamma, \Delta(B))\right. \\
& \left.-\frac{e^{2} \alpha}{\sigma(B, T)}\left[\int_{-\infty}^{\infty} d \omega \frac{\omega-\mu}{T} \frac{1}{4 T \cosh ^{2} \frac{\omega-\mu}{2 T}} \mathcal{A}(\omega, B, \Gamma, \Delta(B))\right]^{2}\right\},
\end{aligned}
$$

where $\mathcal{A}$ is the same function (4.11) as for the electrical conductivity and $\alpha$ is given by Eq. (4.12). Thus we are ready to study the thermal conductivity as a function of $\Gamma, T, \mu, B$, and $\Delta$.

\section{B. Zero magnetic field}

To study the $B=0$ case we substitute the expression for $\mathcal{A}(B=0)$ given by Eq. (4.16) into the general expression for thermal conductivity (5.6).

1. Limit $T \rightarrow 0$

Corresponding to Eq. (4.18) limit $T \rightarrow 0$ is given by

$$
\frac{\kappa}{T}=\frac{\pi^{2}}{3} \alpha \mathcal{A}(\mu, B=0, \Gamma, \Delta) .
$$


Then using Eq. (4.17) for $\mu=\Delta=0$, we obtain

$$
\frac{\kappa(\mu=0)}{T}=\frac{\alpha}{3} .
$$

The factor $\alpha$ in Eq. [5.10) is present also in the DDW 7, 8] and $d S C$ [17] cases where the same expression for the thermal current has been used. The overall numerical factor $1 / 3$ in Eq. (5.10) is the same as in Ref. 17] and it is different from Refs. 7, 8] in the same way as the corresponding factor in expression (4.19) for the electrical conductivity.

\section{Limit $T \ll \Gamma$}

Using the Sommerfeld expansion (4.22) we can also derive next to the leading term in $T^{2}$ to the thermal conductivity for $\Delta=0$

$$
\frac{\kappa}{T}=\frac{\alpha}{3}\left[\mathcal{A}(\omega=\mu, 0, \Gamma, 0)+\frac{7 \pi^{2} T^{2}}{30} \mathcal{A}_{\omega}^{\prime \prime}(\omega=\mu, 0, \Gamma, 0)-\frac{\pi^{2} T^{2}}{9} \frac{\left(\mathcal{A}_{\omega}^{\prime}(\omega=\mu, 0, \Gamma, 0)\right)^{2}}{\mathcal{A}(\omega=\mu, 0, \Gamma, 0)}\right],
$$

where the derivatives of $\mathcal{A}$ are given by Eq. (4.25). For $\mu=0$, since there is no contribution from the thermal power term $\Pi^{E C}$, Eq. (5.11) reduces to the expression

$$
\frac{\kappa(\mu=0)}{T}=\frac{\alpha}{3}\left[1+\frac{7 \pi^{2}}{15} \frac{T^{2}}{\Gamma^{2}}\right], \quad T \ll \Gamma .
$$

\section{3. $\quad$ Limit $T \gg \Gamma$}

For $\Gamma \rightarrow 0$ and $\Delta=0$ substituting Eq. (4.28) into the expression (5.8) for $\kappa$ we obtain (see Appendix C)

$$
\frac{\kappa}{T} \simeq \alpha\left[\frac{\pi}{12} \frac{\mu}{\Gamma}-\frac{\pi^{3}}{36} \frac{T^{2}}{\Gamma \mu}\right], \quad \mu \gg T
$$

where the first term $\sim \mu$ of $\kappa$ arises from the first term of Eq. (5.8) and the second term $\sim 1 / \mu$ originates from the thermal power term $\Pi^{E C}$. Thus, as one would expect, the thermal power contribution into the thermal conductivity is important only for $|\mu| \lesssim T$.

For the case $\mu=\Delta=0$ using Eq. (4.28) we arrive at the expression

$$
\frac{\kappa(\mu=0)}{T}=\frac{\alpha}{\pi}\left[\frac{9 \zeta(3)}{4} \frac{T}{\Gamma}+\frac{\ln 2}{2} \frac{\Gamma}{T}\right], \quad \Gamma \ll T
$$

where $\zeta(z)$ is the Riemann zeta function. Finally using the representation (4.27), which is valid for $\mu=0$ we can get the thermal conductivity for nonzero $\Delta[23]$ :

$$
\frac{\kappa(\mu=0)}{T}=\frac{\alpha}{8 \pi T^{3} \Gamma} \int_{\Delta}^{\infty} d \omega \frac{|\omega|\left(\omega^{2}-\Delta^{2}\right)}{\cosh ^{2} \frac{\omega}{2 T}} \simeq \alpha \frac{\Delta^{2}}{\pi \Gamma T} \exp \left(-\frac{\Delta}{T}\right), \quad \Delta \gg T .
$$

\section{Nonzero magnetic field}

As for the electrical conductivity there are not so many cases available for analytical investigation for $B \neq 0$ and we have to integrate numerically Eq. (5.8) with $\mathcal{A}$ given by Eq. (4.11). There are still a few cases when the analytical treatment is possible and we begin by looking at them.

$$
\text { 1. Limit } T \rightarrow 0
$$

As one can check, Eq. (5.9) is valid even for nonzero $B$, so that

$$
\frac{\kappa}{T}=\frac{\pi^{2}}{3} \alpha \mathcal{A}(\mu, B=0, \Gamma, \Delta), \quad \forall \mu, \quad \Gamma \neq 0 .
$$


Using the narrow width representation (4.14) for $\mathcal{A}$, we arrive at the following result,

$$
\begin{aligned}
\frac{\kappa}{T}=\alpha \frac{\Gamma}{4 \pi T^{3}}\left\{\frac{(e B)^{2}}{2\left[(e B)^{2}+4 \Delta^{2} \Gamma^{2}\right]}\left[\frac{(\Delta+\mu)^{2}}{\cosh ^{2} \frac{\Delta+\mu}{2 T}}+\frac{(\Delta-\mu)^{2}}{\cosh ^{2} \frac{\Delta-\mu}{2 T}}\right]\right. \\
\left.+\sum_{n=1}^{\infty} \frac{2(e B)^{2} n}{(e B)^{2}+4\left(\Delta^{2}+2 e B n\right) \Gamma^{2}}\left[\frac{\left(\sqrt{\Delta^{2}+2 e B n}+\mu\right)^{2}}{\cosh ^{2} \frac{\sqrt{\Delta^{2}+2 e B n}+\mu}{2 T}}+\frac{\left(\sqrt{\Delta^{2}+2 e B n}-\mu\right)^{2}}{\cosh ^{2} \frac{\sqrt{\Delta^{2}+2 e B n}-\mu}{2 T}}\right]\right\}+\frac{\kappa_{1}}{T},
\end{aligned}
$$

where $\kappa_{1}$ is a term originating from the condition of vanishing electrical current. As was discussed above, it is zero for $\mu=0$ and can be neglected for $|\mu| \gg T$. In particular, for $\mu=\Delta=0$ in the strong field limit, $\sqrt{e B} \gtrsim 4 T$ we obtain from Eq. (5.17) the expression

$$
\frac{\kappa}{T}=\alpha \frac{8 \Gamma e B}{\pi T^{3}} e^{-\frac{\sqrt{e B}}{T}}
$$

\section{Numerical calculation of thermal conductivity}

In Figs. 7 and 8 we present the results for the thermal conductivity that were obtained for the same values of the model parameters as the data for electrical conductivity shown in Figs. 3 and 4 Comparing these figures, one can see that the behavior of the thermal conductivity is rather similar to the behavior of electrical conductivity and to see more subtle differences we have to consider the temperature dependence of the Lorenz number as done in Sec. VI]

It is also interesting to investigate numerically the contribution from the second term of Eq. (5.8) to the thermal conductivity. This is done in Fig. [9] where one can clearly see that when the temperature grows and $T$ becomes $\lesssim|\mu|$ the second term of Eq. (5.8) makes an important negative contribution to the thermal conductivity.

In Figs. [10 and 11] we present the results for the dependence of thermal conductivity on the magnetic field $B$ that were obtained for the same values of the model parameters as the data for electrical conductivity shown in Figs. 5 and 6

\section{WIEDEMANN-FRANZ LAW}

After deriving in Secs. IV and $\mathbf{V}$ the expressions for electrical and thermal conductivities in the various limits we are ready to consider their implications for the WF law.

\section{A. Limit $T \rightarrow 0$, arbitrary field, and chemical potential}

For finite $\Gamma$, arbitrary $\mu, B$, and $\Delta$ in the limit $T \rightarrow 0$ one can see from Eq. (4.31) [see also Eq. (4.18) for $B=0$ ] and (5.16) [see also Eq. (5.9) for $B=0$ ] that WF law is maintained:

$$
L_{0}=\frac{\kappa}{\sigma T}=\frac{\pi^{2}}{3} \frac{k_{B}^{2}}{e^{2}}
$$

This result can be understood from the qualitative arguments given, for example, in Ref. [4]. The expressions for the electrical, Eq. (4.10), and thermal, Eq. (5.8), conductivities calculated within the bare bubble approximation are very similar in the limit $T \rightarrow 0$ when the second term of Eq. (5.8) vanishes. Both expressions contain the function $\mathcal{A}(\omega)$ multiplied by the derivative of the Fermi distribution $f(\omega)=-n_{F}^{\prime}(\omega-\mu)$, with the only difference that the thermal conductivity is also multiplied by the factor $\sim(\omega-\mu)^{2}$. These prefactors $f(\omega)$ and $g(\omega)=-(\omega-\mu)^{2} n_{F}^{\prime}(\omega-\mu) / T^{2}$ are shown in Figs. 12 and 13. When the temperature $T$ goes to zero, the $\delta$-like spikes of $f(\omega)$ and $g(\omega)$ occur at the same value $\omega=\mu$ and the WF law is maintained.

\section{B. Zero magnetic field at $T \neq 0$}

For $\mu=0$ and $T \gg \Gamma$ considering Eqs. (5.14) and (4.29) we obtain that the WF law is violated,

$$
L=\frac{\kappa}{\sigma T}=\frac{9 \zeta(3)}{2 \ln 2} \frac{k_{B}^{2}}{e^{2}} \simeq 2.37 L_{0},
$$


while for $\mu \gg T$ Eqs. (5.13) and (4.29) show that the Lorenz number has its usual value

$$
L=L_{0} .
$$

These two results can also be understood qualitatively using Figs. 12 and 13 For $|\mu| \gg T>0$ the electrical conductivity is determined by energies $\omega \approx \mu=0$, while the thermal conductivity is determined by the energies near $\omega \approx \mu \pm k_{B} T$. If the function $\mathcal{A}(\omega)$ does not vary appreciably over the energy range $\mu-k_{B} T$ to $\mu+k_{B} T$, the Lorenz number may still be $\sim 1$ even for finite $T, T \ll \mu$, as follows from Eq. (6.3). If $\mu$ becomes of the same order of $T$, the dimensionless values $\mu / T$ and $\mu / T \pm 1$ are apart and the Lorenz number deviates from 1 as seen from Eq. (6.2).

For nonzero $\Delta \gg T$ and $\mu=0$ we get from Eqs. (4.30) and (5.15) that

$$
L=\frac{k_{B}^{2}}{e^{2}} \frac{\Delta^{2}}{T^{2}}
$$

i.e., $L>L_{0}$ for $\Delta>(\pi / \sqrt{3}) T$. This dominance of the thermal conductivity can be easily understood from the fact that when the gap opens, the electrical conductivity diminishes more strongly because it is determined by the energies $\omega \approx \mu$ near the Fermi surface.

\section{Nonzero magnetic field at $T \neq 0$}

For $\mu=\Delta=0$ in the strong field limit, $\sqrt{e B} \gtrsim 4 T$ from Eqs. (4.33) and (5.18) we obtain that the Lorenz number becomes field dependent:

$$
L=\frac{32 e B}{T^{2}} e^{-\frac{\sqrt{e B}}{T}} .
$$

In the presence of magnetic field the function $\mathcal{A}(\omega)$ (see Fig. 14) varies appreciably over the energy range $\omega-k_{B} T$ to $\omega+k_{B} T$, so that it is nonzero for $\omega=0$ and very small at $\omega= \pm k_{B} T$. This feature of $\mathcal{A}(\omega)$, along with the small values of $\mu$, can produce rather strong violation of the WF law.

Our qualitative arguments are indeed confirmed by Figs. [15] and [16 where we show the temperature dependence of $L(T)$ at half-filling (Fig. 15) and away from it (Fig. 16). Both these figures are computed on the basis of Eqs. (4.10), (5.8), and (4.11). For $\mu=0$ the value of $L$ always goes down from $L_{0}$ as the temperature increases, then $L$ increases crossing $L_{0}$, and finally it goes to its zero-field value (6.2). One can see from Fig. 16] that for nonzero $\mu$ the deviations of $L(T)$ from $L_{0}$ become less pronounced (see also the discussion of Fig. (17).

It is also instructive to investigate the importance of the second term of thermal conductivity, Eq. (5.8), for the Lorenz number calculated with and without this term (as already done in Fig. 9 for the thermal conductivity itself). These results are given in Fig. 17 and they show that the second term of thermal conductivity is indeed crucial to get the correct answer. Note that for the case of nonzero $\mu$ the situation with the WF law is more complicated because the value of $\mu$ may coincide with $\omega$, corresponding to the maximum of $\mathcal{A}(\omega)$. In this case $L(T)$ will firstly increase, as one can see in Fig. [17 for $B=12 \mathrm{~T}$.

\section{CONCLUSIONS}

Let us compare the results derived in the present paper with the experimental results obtained in Ref. [1]. It is still unknown whether the DDW state exists and/or plays an important role in the electron doped compound used in Ref. 1], so that making this comparison would help to address these questions. First, we observe that while the results of [1] suggest that the WF law is violated at $T \rightarrow 0$, there is no violation of the WF law in this limit in the DDW scenario of the pseudogap. Since in Fig. 3 of Ref. [1] the electrical conductivity is a constant, the line $\kappa_{e}(T) / T$ directly represents the normalized Lorenz number $L(T) / L_{0}$. For finite temperatures there is then some similarity between Fig. 15] (or Fig. 16] for $\mu \neq 0$ case) and Fig. 3 of Ref. [1] where as $T$ increases the thermal conductivity crosses from the region with $\kappa_{e} / T<L_{0} / \rho_{0}$ to the region with $\kappa_{e}(T) / T>L_{0} / \rho_{0}$ resembling the character of the WF law violation seen in the experiment.

However, in the experiment, the electrical conductivity is flat while the thermal conductivity changes significantly in a subkelvin range. On the other hand, theoretical calculations show that the electrical (see Figs. 3 and 4) and thermal (see Figs. (7) and 8) conductivities vary simultaneously in a range of $10 \mathrm{~K}$. Note that the wider range of temperatures can be probably attributed to the fact that the values of the model parameters we took for the numerical estimates are more appropriate for the hole-doped compounds. 
It is obvious from Fig. 15 that such a behavior of $L(T)$ is due to the presence of the magnetic field. This confirms our claim that to interpret theoretically the experiment in Ref. [1] one should take into account the influence of the external field.

Our main results can be summarized as follows.

(1) We have obtained analytical expressions for electrical conductivity (4.10) and thermal conductivity (5.8) in the DDW state in presence of an external magnetic field.

(2) We have established a correspondence between the expression for the electrical conductivity (4.3) in the DDW state written in terms of the generalized velocity $\mathbf{V}(\mathbf{k})$, Eq. [2.15), and the dc conductivity derived by Langer [28] in the bubble approximation with the vertex (4.4).

(3) We have shown that in the DDW system in the presence of impurities the WF law holds in $T \rightarrow 0$ limit for an arbitrary field $B$ and chemical potential $\mu$. This is checked within the bubble approximation, i.e., not including the impurity vertex. The influence of the impurity vertex on the properties of the DDW state in zero field at half-filling can be considered using the heuristical arguments of Durst and Lee 17] for $d \mathrm{SC}$ state. Since in the $d \mathrm{SC}$ state the thermal (and spin) currents are proportional to the group velocity, they can relax through either intranode scattering or scattering between nodes. As a result, the different types of scattering play nearly the same role and therefore vertex corrections do not contribute to the thermal (and spin) conductivity. This is not the case of the electrical current in the $d \mathrm{SC}$ state, because it depends only on the Fermi velocity. Thus, the electrical current can relax more effectively via scattering from node to node than it can via scattering within a single node. This difference is taken into account by considering vertex corrections which modify the bare bubble expression for the electrical conductivity. Since the electrical current in the DDW state is also proportional to the group velocity, the corresponding impurity vertex should not contribute to the electrical conductivity. Moreover, the thermal current in the DDW state is also proportional to the group velocity, so that one can rely on the same arguments about the impurity vertex as for the $d \mathrm{SC}$ case. Thus we do not expect that in the DDW state the impurity vertex can be a source of the WF violation at $T \rightarrow 0$. These observations should be confirmed by detailed calculations of the impurity vertex corrections, which, however, are beyond the scope of the present paper.

(4) For finite temperatures $T \lesssim|\mu|$, the WF law violation is possible and in zero field the thermal conductivity dominates over the electrical conductivity, i.e., $L(T) / L_{0}>1$.

(5) For $T \lesssim|\mu|$ in the nonzero field the WF law violation becomes even stronger than in zero field and depending on the temperature both regimes $L(T) / L_{0} \ll 1$ and $L(T) / L_{0}>1$ are possible.

(6) For $T \ll|\mu|$ there is no WF violation even in the presence of magnetic field.

Finally, we would like to stress that the results of the present paper should be applicable not only to the DDW state, but to a wider class of theories. Here we started from the DDW Hamiltonian (2.1) and, to simplify the problem, approximated it by the $\mathrm{QED}_{3}$ Lagrangian (2.38). There is, however, a number of systems, e.g., pyrolitic graphite 24], that can also be described by the Lagrangian (2.38), so that our results would also be relevant for them.

\section{ACKNOWLEDGMENTS}

We gratefully acknowledge A. Gröger for a stimulating discussion. S.G.Sh would like to thank N. Andrenacci, L. Benfatto and L. Carlevaro for helpful discussions and W. Kim for useful correspondence. This work was supported by Research Project No. 20-65045.01 of the Swiss NSF. The work of V.P.G. was supported by the SCOPES Projects No. 7UKPJ062150.00/1 and No. 7 IP 062607 of the Swiss NSF and by Grant No. PHY-0070986 of NSF (USA).

\section{APPENDIX A: NODAL FERMION GREEN'S FUNCTION IN AN EXTERNAL MAGNETIC FIELD}

In the coordinate space, the fermion propagator has the following form in the proper-time representation

$$
S(x, y)=(i \hat{D}+\Delta)_{x}\left\langle x\left|\frac{-1}{\Delta^{2}+\hat{D}^{2}}\right| y\right\rangle=-i(i \hat{D}+\Delta)_{x} \int_{0}^{\infty} d s\left\langle x\left|\exp \left[-i s\left(\Delta^{2}+\hat{D}^{2}\right)\right]\right| y\right\rangle,
$$

where $\hat{D}=\gamma^{\nu} D_{\nu}$ and the "long" derivative $D_{\nu}$ is given by Eq. (2.37). The matrix element $\left\langle x\left|\exp \left[-i s\left(\Delta^{2}+\hat{D}^{2}\right)\right]\right| y\right\rangle$ can be calculated using the Schwinger (proper time) approach 22] (see also a pedagogical overview in Ref. [19]). The main idea of this method is based on the interpretation of $\left\langle x\left|\exp \left[-i s \hat{D}^{2}\right]\right| y\right\rangle$ as the coordinate representation of the proper-time "evolution operator" $U(s)=\exp [-i H s]$, where we have introduced the "Hamiltonian"

$$
H=\hat{D}^{2}=D^{2}-\frac{e}{2} \sigma^{\rho \nu} F_{\rho \nu}, \quad F_{\rho \nu}=\partial_{\rho} A_{\nu}-\partial_{\nu} A_{\rho}, \quad \sigma^{\rho \nu}=\frac{i}{2}\left[\gamma^{\rho}, \gamma^{\nu}\right]
$$


The matrix element of the evolution operator can be evaluated [19], for example, using either operator or functional integral formalism:

$$
\langle x|U(s)| y\rangle=\frac{e^{-i \pi / 4}}{8(\pi s)^{3 / 2}} \exp \left[i e \int_{y}^{x} d \xi_{\nu} A^{\nu}(\xi)+\frac{i}{4}(x-y) f(s)(x-y)-L(s)+i \frac{e}{2} \sigma \mathbb{F} s\right],
$$

with

$$
f(s) \equiv e \mathbb{F} \operatorname{coth}(e \mathbb{F} s), \quad L(s) \equiv \frac{1}{2} \operatorname{tr} \ln \left(\frac{\sinh e \mathbb{F} s}{e \mathbb{F} s}\right),
$$

where we have used matrix notations, e.g., $F_{\rho \nu} \equiv(\mathbb{F})_{\rho \nu}$ and the integral with $A_{\nu}$ is calculated along the straight line. This operator differs from the four-dimensional version of $\langle x|U(s)| y\rangle$ [19] in the power dependence of the proper time and in the numerical prefactors. Plugging (A3) into (A1) one obtains

$$
\begin{aligned}
S(x, y) & =\exp \left(i e \int_{y}^{x} d \xi_{\nu} A^{\nu}(\xi)\right) \frac{e^{-i 3 \pi / 4}}{8 \pi^{3 / 2}} \int_{0}^{\infty} \frac{d s}{s^{3 / 2}}\left[\Delta-\frac{1}{2} \gamma^{\rho}[f(s)+e \mathbb{F}]_{\rho \nu}(x-y)^{\nu}\right] \\
& \times \exp \left[-i \Delta^{2} s+\frac{i}{4}(x-y) f(s)(x-y)-L(s)+i \frac{e}{2} \sigma \mathbb{F} s\right] .
\end{aligned}
$$

Finally, to perform calculations for the case of interest one should evaluate $f(s), L(s)$ and $\exp [i(e / 2) \sigma \mathbb{F} s]$ for $\mathbb{F}$ containing purely magnetic background field $B$. The corresponding expressions for these values are

$$
\begin{aligned}
& f(s)_{\rho \nu}=-\frac{1}{s}\left[g_{\rho \nu}+\frac{\left(\mathbb{F}^{2}\right)_{\rho \nu}}{B^{2}}(1-e B s \cot (e B s))\right], \\
& \exp [-L(s)]=\frac{e B s}{\sin e B s}, \\
& \exp \left[i \frac{e}{2} \sigma \mathbb{F} s\right]=\cos e B s+\gamma^{1} \gamma^{2} \sin e B s .
\end{aligned}
$$

In the Matsubara frequency-momentum representation this leads to Eq. (3.2).

It is convenient to write down an alterative form of the fermion propagator in a magnetic field as a sum over the Landau-level poles. In deriving it we follow Refs. 21, 25]. Introducing the shorthand notations we rewrite Eq. (3.2) as follows,

$$
\tilde{S}(i \omega, \mathbf{p})=-\int_{0}^{\infty} d s \exp \left[-s\left(a+\mathbf{p}^{2} \frac{\tanh (e B s)}{e B s}\right)\right] \times[(b+c \tanh (|e B s|))(1+d \tanh (|e B s|))],
$$

where

$$
\begin{aligned}
& a=\Delta^{2}-(i \omega)^{2}, \quad b=i \omega \gamma^{0}-p_{1} \gamma^{1}-p_{2} \gamma^{2}+\Delta, \\
& c=-i\left(p_{2} \gamma^{1}-p_{1} \gamma^{2}\right) \operatorname{sgn}(e B), \quad d=-i \gamma^{1} \gamma^{2} \operatorname{sgn}(e B) .
\end{aligned}
$$

Using the identity $\tanh x=1-2 \exp (-2 x) /[1+\exp (-2 x)]$, the relation 32]

$$
(1-z)^{-(\alpha+1)} \exp \left(\frac{x z}{z-1}\right)=\sum_{n=0}^{\infty} L_{n}^{\alpha}(x) z^{n}, \quad|z|<1,
$$

with $z=-\exp (-2|e B| s), x=2 \frac{\mathbf{p}^{2}}{|e B|}$, we arrive at the expression

$$
\begin{aligned}
& \tilde{S}(i \omega, \mathbf{p})=-\exp \left(-\frac{\mathbf{p}^{2}}{|e B|}\right) \\
& \times \int_{0}^{\infty} d s \exp \left[-s a+\frac{2 \mathbf{p}^{2}}{|e B|} \frac{z}{z-1}\right]\left[(b+c)(1+d)+(4 c d+2 c+2 b d) \frac{z}{1-z}+4 c d \frac{z^{2}}{(1-z)^{2}}\right] .
\end{aligned}
$$


Then using the identity $L_{n}^{\alpha-1}(x)=L_{n}^{\alpha}(x)-L_{n-1}^{\alpha}(x)$ and the definitions $L_{n} \equiv L_{n}^{0}, L_{-1}^{\alpha}=0$ we obtain

$$
\begin{aligned}
& \tilde{S}(i \omega, \mathbf{p})=-\exp \left(-\frac{\mathbf{p}^{2}}{|e B|}\right) \int_{0}^{\infty} d s \exp (-s a)\left[(b+c)(1+d) \sum_{n=0}^{\infty} L_{n}(x) z^{n}\right. \\
& \left.+(-(b+c)(1+d)+2 c+2 b d) \sum_{n=0}^{\infty} L_{n-1}(x) z^{n}+4 c d \sum_{n=0}^{\infty} L_{n-1}^{1}(x) z^{n}\right] \\
& =-\exp \left(-\frac{\mathbf{p}^{2}}{|e B|}\right) \int_{0}^{\infty} d s \exp (-s a)\left\{\left(\Delta+i \omega \gamma^{0}\right)\left[\left(1-\operatorname{sgn}(e B) i \gamma^{1} \gamma^{2}\right) \sum_{n=0}^{\infty} L_{n}(x) z^{n}-\left(1+\operatorname{sgn}(e B) i \gamma^{1} \gamma^{2}\right) \sum_{n=0}^{\infty} L_{n-1}(x) z^{n}\right]\right. \\
& \left.\quad+4\left(p_{1} \gamma^{1}+p_{2} \gamma^{2}\right) \sum_{n=0}^{\infty} L_{n-1}^{1}(x) z^{n}\right\} .
\end{aligned}
$$

Finally, integrating over $s$ we arrive at Eq. (3.3).

\section{APPENDIX B: GENERALIZED POLARIZATION BUBBLE AND NODAL APPROXIMATION}

The calculations of electrical, thermal, and even spin conductivities are quite similar for both DDW and $d \mathrm{SC}$ [17] nodal systems. Thus instead of repeating the same calculation several times and to underline the similarities and differences between DDW and $d \mathrm{SC}$ cases, it is rather convenient to define a generalized polarization tensor $\Pi^{g g^{\prime}}$ that depends on the generalized coupling parameters $g, g^{\prime}$

$$
g, g^{\prime}=\left[e,\left\{\begin{array}{l}
i \omega+i \Omega / 2 \\
\omega+\Omega / 2-\mu
\end{array}\right]\right.
$$

Two lines in the definition of $g, g^{\prime}$ correspond to the Matsubara and real frequencies, respectively, and the origin of the chemical potential $\mu$ in the second line will become clear later. The generalized bubble is

$$
\Pi_{\alpha \beta}^{g g^{\prime}}(i \Omega)=2 T \int_{\mathrm{RBZ}} \frac{d^{2} k}{(2 \pi)^{2}} \sum_{i \omega} g g^{\prime} \operatorname{tr}\left[G(i \omega, \mathbf{k}) V_{\alpha}(\mathbf{k}) G(i \omega+i \Omega, \mathbf{k}) V_{\beta}(\mathbf{k})\right],
$$

where $G(i \omega, \mathbf{k})$ is the Green's function (2.20), but evaluated in the external field, and $\mathbf{V}(\mathbf{k})$ is generalized velocity given by Eq. (2.15). The integral is over the reduced Brillouin zone (RBZ) and the factor 2 before the integral is due to the spin degree of freedom $s$. To compare this expression with $d$ SC case [see Eq. (A1) of Ref. [17]] we note that the summation over the spin degree of freedom is already included in the Nambu formalism.

Using the spectral representation for the fermion Green's function

$$
G(i \omega+\mu, \mathbf{k})=\int_{-\infty}^{\infty} \frac{A\left(\omega_{1}, \mathbf{k}\right)}{i \omega+\mu-\omega_{1}} d \omega_{1}
$$

where $A\left(\omega_{1}, \mathbf{k}\right)$ is the spectral density, we arrive at

$$
\Pi_{\alpha \beta}^{g g^{\prime}}(i \Omega)=2 \int_{\mathrm{RBZ}} \frac{d^{2} k}{(2 \pi)^{2}} \int d \omega_{1} \int d \omega_{2} \operatorname{tr}\left[A\left(\omega_{1}, \mathbf{k}\right) V_{\alpha}(\mathbf{k}) A\left(\omega_{2}, \mathbf{k}\right) V_{\beta}(\mathbf{k})\right] R
$$

with

$$
R=T \sum_{i \omega} g g^{\prime} \frac{1}{i \omega+\mu-\omega_{1}} \frac{1}{i \omega+\mu+i \Omega-\omega_{2}} .
$$

Since the intermediate results differ depending on the frequency dependence of the coupling parameters $g, g^{\prime}$, we consider the frequency-independent and frequency-dependent couplings separately. For $g=g^{\prime}=e$ (frequency independent coupling), evaluating the sum and then continuing $i \Omega \rightarrow \Omega+i 0$ we get

$$
R=g^{2} \frac{n_{F}\left(\omega_{1}-\mu\right)-n_{F}\left(\omega_{2}-\mu\right)}{\omega_{1}-\omega_{2}+\Omega+i 0}=\frac{g^{2}}{2} \frac{\tanh \frac{\omega_{2}-\mu}{2 T}-\tanh \frac{\omega_{1}-\mu}{2 T}}{\omega_{1}-\omega_{2}+\Omega+i 0} .
$$


For the frequency-dependent coupling $g=g^{\prime}=i \omega+i \Omega / 2$ the evaluation of the Matsubara sum (B55) gives 33]

$$
R=T \sum_{i \omega} \frac{(i \omega+i \Omega / 2)^{2}}{\left(i \omega+\mu-\omega_{1}\right)\left(i \omega+\mu+i \Omega-\omega_{2}\right)}=\frac{\left(\omega_{1}-\mu+i \Omega / 2\right)^{2} n_{F}\left(\omega_{1}-\mu\right)-\left(\omega_{2}-\mu-i \Omega / 2\right)^{2} n_{F}\left(\omega_{2}-\mu\right)}{\omega_{1}-\omega_{2}+i \Omega} .
$$

Then, continuing to real frequencies $i \Omega \rightarrow \Omega+i 0$ we obtain

$$
\operatorname{Im} R=\frac{\pi}{2}\left(\omega_{1}-\mu+\Omega / 2\right)^{2}\left[\tanh \frac{\omega_{1}-\mu}{2 T}-\tanh \frac{\omega_{1}-\mu+\Omega}{2 T}\right] \delta\left(\omega_{1}-\omega_{2}+\Omega\right) .
$$

Comparing the imaginary part of Eq. (B6) with Eq. (B8) one can see that the latter equation can be obtained from the former by the direct substitution of $g=g^{\prime}=\omega+\Omega / 2-\mu$. This explains the difference between the upper and lower lines in Eq. B1).

Similarly, evaluating the Matsubara sum (B5) with $g=e, g^{\prime}=i \omega+i \Omega / 2$ we get

$$
R=T \sum_{i \omega} \frac{e(i \omega+i \Omega / 2)}{\left(i \omega+\mu-\omega_{1}\right)\left(i \omega+\mu+i \Omega-\omega_{2}\right)}=\frac{e\left(\omega_{1}-\mu+i \Omega / 2\right) n_{F}\left(\omega_{1}-\mu\right)-e\left(\omega_{2}-\mu-i \Omega / 2\right) n_{F}\left(\omega_{2}-\mu\right)}{\omega_{1}-\omega_{2}+i \Omega},
$$

so that

$$
\operatorname{Im} R=\frac{\pi}{2} e\left(\omega_{1}-\mu+\Omega / 2\right)\left[\tanh \frac{\omega_{1}-\mu}{2 T}-\tanh \frac{\omega_{1}-\mu+\Omega}{2 T}\right] \delta\left(\omega_{1}-\omega_{2}+\Omega\right) .
$$

Finally, we obtain for the imaginary part of the tensor polarization $\Pi_{R}^{g g^{\prime}}(\Omega+i 0)$ the following expression

$$
\operatorname{Im} \Pi_{\alpha \beta}^{g g^{\prime}}(\Omega+i 0)=\pi \int_{\mathrm{RBZ}} \frac{d^{2} k}{(2 \pi)^{2}} \int_{-\infty}^{\infty} d \omega g g^{\prime}\left[\tanh \frac{\omega-\mu}{2 T}-\tanh \frac{\omega-\mu+\Omega}{2 T}\right] \operatorname{tr}\left[A(\omega, \mathbf{k}) V_{\alpha}(\mathbf{k}) A(\omega+\Omega, \mathbf{k}) V_{\beta}(\mathbf{k})\right] .
$$

Looking at Eqs. (B2) and (B11) one can notice that the Green's function $G$ and the associated spectral density $A$ in the external field are in fact unknown. Instead of considering these functions, we have constructed in Sec. III the Green's function (3.3) for the linearized nodal Lagrangian (2.38) that is valid in the vicinity of the four nodal points. Thus, in Eq. (B2) we replace the integration over the reduced Brillouin zone by the integral over the $\mathbf{k}$-space surrounding each node and sum over the four nodal subzones:

$$
\int_{\mathrm{RBZ}} \frac{d^{2} k}{(2 \pi)^{2}} \rightarrow \frac{1}{2} \sum_{j=1}^{4} \int \frac{d k_{x} d k_{x}}{(2 \pi)^{2}} \rightarrow \frac{1}{2} \sum_{j=1}^{4} \int \frac{d^{2} p}{(2 \pi)^{2} v_{F} v_{D}}=\frac{1}{4 \pi v_{F} v_{D}} \sum_{j=1}^{4} \int_{0}^{p_{0}} p d p \int_{0}^{2 \pi} \frac{d \theta}{2 \pi},
$$

where $p_{1}=v_{F} k_{x}=p \cos \theta, p_{2}=v_{D} k_{y}=p \sin \theta, p=\sqrt{p_{1}^{2}+p_{2}^{2}}, p_{0}=\sqrt{\pi v_{F} v_{D}} /(2 a)$ and the local nodal coordinate systems $\left(k_{x}, k_{y}\right)$ are shown in Fig. 2] Note again that, comparing with [17], an extra factor 1/2 appears due to the fact that the original integral is over the reduced Brillouin zone.

The advantage of the scaled variables $p_{1}$ and $p_{2}$ is that they correspond to the "relativistic" case $v_{F}=v_{D}=c=1$. Moreover, we can use the spectral function $A_{D}$ in Eq. (3.6) inside the integral over $p$, so that all necessary factors with $v_{F}$ and $v_{D}$ are already outside the integral (B12). We should only provide the rule that allows us to restore the model parameters coming along with the magnetic field in the final result [34]: $e B \rightarrow\left(\hbar v_{F} v_{D} / c\right) e B$.

We replace the generalized velocities $\mathbf{V}(\mathbf{k})$ in Eq. (B11) by their values $\mathbf{V}_{\mathbf{k}=\mathbf{N}}$ on the Fermi surface at half-filling $(\mu=0)$. Note that for the DDW case this approximation is more severe than for the $d$ SC case because for $\mu \neq 0$ the true Fermi surface does not coincide with the Fermi surface at half-filling. This approximation puts some restrictions on the values of $\mu$, so that we cannot move far away from half-filling. The bubble (B11) takes the form

$\operatorname{Im} \Pi_{\alpha \beta}^{g g^{\prime}}(\Omega+i 0)=\frac{\pi}{2 v_{F} v_{D}} \sum_{j=1}^{4} \int \frac{d^{2} p}{(2 \pi)^{2}} \int_{-\infty}^{\infty} d \omega g g^{\prime}\left[\tanh \frac{\omega-\mu}{2 T}-\tanh \frac{\omega-\mu+\Omega}{2 T}\right] \operatorname{tr}\left[A_{D}(\omega, \mathbf{p}) \gamma^{0} V_{\alpha} A_{D}(\omega+\Omega, \mathbf{p}) \gamma^{0} V_{\beta}\right]$,

where the spectral density (3.6) includes the broadening of the spectral lines (3.8) due to impurities and we inserted the $\gamma^{0}$ matrix [see Eq. (3.9)]. Note that in Eq. (B3) we used only the translationary invariant part (3.2) of the Green's function (3.1) calculated in the external field, since its translation non-invariant part cancels out when substituted in the bubble $\Pi$. 
Since we are interested only in the longitudinal conductivities and because the system is isotropic, we can define the longitudinal polarization function $\Pi^{g g^{\prime}}$ as follows (the sum over dummy index is implied):

$$
\Pi^{g g^{\prime}}(\Omega) \equiv \frac{1}{2} \Pi_{\alpha \alpha}^{g g^{\prime}}(\Omega)
$$

Then evaluating the sum over nodes in Eq. (B13) by using the identities

$$
\sum_{j=1}^{4} v_{l \alpha}^{(j)} v_{l \beta}^{(j)}=2 v_{l}^{2} \delta_{\alpha \beta}, \quad \sum_{j=1}^{4} v_{l \alpha}^{(j)} v_{l^{\prime} \beta}^{(j)}=2 v_{l} v_{l^{\prime}} \epsilon_{l l^{\prime}} \epsilon_{\alpha \beta} \quad\left(l \neq l^{\prime}\right), \quad \mathbf{v}_{l} \equiv\left\{\mathbf{v}_{F}, \mathbf{v}_{D}\right\}
$$

with $\epsilon_{i j}$ being antisymmetric tensor, we arrive at the final result

$$
\begin{aligned}
& \operatorname{Im}^{g g^{\prime}}(\Omega+i 0)=\frac{\pi}{v_{F} v_{D}} \int \frac{d^{2} p}{(2 \pi)^{2}} \int_{-\infty}^{\infty} d \omega g g^{\prime}\left[\tanh \frac{\omega-\mu}{2 T}-\tanh \frac{\omega-\mu+\Omega}{2 T}\right] \\
& \times\left(v_{F}^{2} \operatorname{tr}\left[A_{D}(\omega, \mathbf{p}) \gamma^{1} A_{D}(\omega+\Omega, \mathbf{p}) \gamma^{1}\right]+v_{D}^{2} \operatorname{tr}\left[A_{D}(\omega, \mathbf{p}) \gamma^{2} A_{D}(\omega+\Omega, \mathbf{p}) \gamma^{2}\right]\right) .
\end{aligned}
$$

Since both the electrical current (2.14) and thermal current (2.18) have two terms, a "Fermi" term proportional to $\mathbf{v}_{F}$ and $\sigma_{3}$ and a "gap" term proportional to $\mathbf{v}_{D}$ and $\sigma_{2}$, our calculation of the current-current polarization function (B2) is in fact similar to the calculation of the thermal current bubble for the $d$ SC case done in Ref. [17]. Evaluating the polarization function (B13) we obtain four bubbles: Fermi-Fermi, Fermi-gap, gap-Fermi, and gap-gap. However, since $\mathbf{v}_{F} \perp \mathbf{v}_{D}$ at each of the gap nodes, the two cross terms cancel, as reflected in the second identity in Eq. (B15). Therefore both electrical and thermal longitudinal conductivities have two terms: the Fermi term with the velocity $v_{F}^{2}$ and the gap term with the velocity $v_{D}^{2}$. Thus, finally we approximated the polarization bubble for the Hamiltonian (2.1) by the polarization bubble for the nodal Lagrangian (2.38).

\section{APPENDIX C: SOME INTEGRALS}

Here we derive the expression (5.13) for $\kappa$. It follows from the general expression (5.8) with $\mathcal{A}$ given by Eq. (4.28). The corresponding integrals are easily evaluated in terms of polylogarithmic functions, $\operatorname{Li}_{n}(z),[35]$ :

$$
\begin{aligned}
J & =\int_{-\infty}^{\infty} \frac{d \omega(\omega-\mu)^{2}|\omega|}{\cosh ^{2}((\omega-\mu) / 2 T)} \\
& =8 T^{2} \mu^{2} \ln (2 \cosh (\mu / 2 T))+16 T^{3} \mu\left[\operatorname{Li}_{2}\left[-e^{\mu / T}\right]-\operatorname{Li}_{2}\left[-e^{-\mu / T}\right]\right]-24 T^{4}\left[\operatorname{Li}_{3}\left[-e^{\mu / T}\right]+\operatorname{Li}_{3}\left[-e^{-\mu / T}\right]\right]
\end{aligned}
$$

and

$$
I=\int_{-\infty}^{\infty} \frac{d \omega(\omega-\mu)|\omega|}{\cosh ^{2}((\omega-\mu) / 2 T)}=8 T^{3}\left[\operatorname{Li}_{2}\left[-e^{-\mu / T}\right]-\operatorname{Li}_{2}\left[-e^{\mu / T}\right]\right]-8 T^{2} \mu \ln (2 \cosh (\mu / 2 T))
$$

These rather complicated expressions for $I$ and $J$ can be expressed via elementary functions in the limit $\mu \gg T$. We begin with the more simple case of $I$. Using the identity [35]

$$
\operatorname{Li}_{2}(z)=-\mathrm{Li}_{2}(1 / z)-\frac{1}{2} \ln ^{2}(-z)-\frac{\pi^{2}}{6}, \quad z \notin(0,1), \quad \operatorname{Li}_{2}(0)=0
$$

we obtain that in the limit $\mu \gg T$

$$
\operatorname{Li}_{2}\left[-e^{-\mu / T}\right]-\operatorname{Li}_{2}\left[-e^{\mu / T}\right]=2 \operatorname{Li}_{2}\left(-e^{-\mu / T}\right)+\frac{1}{2} \ln ^{2}\left(e^{\mu / T}\right)+\frac{\pi^{2}}{6} \simeq \frac{\mu^{2}}{2 T^{2}}+\frac{\pi^{2}}{6} .
$$

Thus the final expression for $I$ reads as

$$
I \simeq \frac{4 \pi^{2}}{3} T^{3}, \quad \mu \gg T
$$


To simplify $J$, in addition to Eq. (C3), we use the corresponding identity for $\operatorname{Li}_{3}(z)$ [35]:

$$
\operatorname{Li}_{3}(z)=\operatorname{Li}_{3}(1 / z)-\frac{1}{6} \ln ^{3}(-z)-\frac{\pi^{2}}{6} \ln (-z), \quad z \notin(0,1), \quad \operatorname{Li}_{3}(0)=0
$$

and for $\mu \gg T$ we obtain that

$$
J \simeq \frac{4 \pi^{2}}{3} T^{3} \mu, \quad \mu \gg T .
$$

Putting Eqs. (C7) and (C5) along with Eq. (4.29) into Eq. (5.8), we arrive at the final result (5.13).

[1] R.W. Hill, C. Proust, L. Taillefer, P. Fournier, and R.L. Greene, Nature 414, 711 (2001).

[2] C. Proust, E. Boaknin, R.W. Hill, L. Taillefer, and A.P. Mackenzie, Phys. Rev. Lett. 89, 147003 (2002).

[3] J.S. Langer, Phys. Rev. 128, 110 (1962).

[4] P.L. Taylor, A Quantum Approach to the Solid State, (Prentice-Nall, New Jersey, 1970).

[5] L. Smrčka and P. Středa, J. Phys. C: Solid State Phys. 10, 2153 (1977).

[6] A. Houghton, S. Lee, J.B. Marston, Phys. Rev. B 65, 220503 (2002).

[7] X. Yang and C. Nayak, Phys. Rev. B 65, 064523 (2002).

[8] W. Kim and J.P. Carbotte, Phys. Rev. B 66, 033104 (2002).

[9] S. Chakravarty, R.B. Laughlin, D.K. Morr, and C. Nayak, Phys. Rev. B 63, 094503 (2001).

[10] T. Timusk and B. Statt, Rep. Progr. Phys. 62, 61 (1999).

[11] C. Nayak, Phys. Rev. B 62, 4880 (2000).

[12] J.L. Tallon and J.W. Loram, Physica C 349, 53 (2001).

[13] O. Vafek, A. Melikyan, Z. Tešanović, Phys. Rev. B 64, 224508 (2001).

[14] A.S. Mel'nikov in Vortices in Unconventional Superconductors and Superfluids, edited by R.P. Huebener, N. Schopohl and G.E. Volovik (Springer, Berlin, 2002), Chap. 12.

[15] A.A. Nersesyan and G.E. Vachanadze, J. Low Temp. Phys. 77, 293 (1989).

[16] C.P. Enz, A Course on Many-Body Theory Applied to Solid-State Physics, (World Scientific, Singapore, 1992).

[17] A.C. Durst and P.A. Lee, Phys. Rev. B 62, 1270 (2000).

[18] D. Morr, Phys. Rev. Lett. 89, 106401 (2002).

[19] W. Dittrich, H. Geis, Probing the Quantum Vacuum: Perturbative Effective action approach in Quantum Electrodynamics and its Application, Springer, 2000.

[20] R. Jackiw and S. Templeton, Phys. Rev. D 23, 2291 (1981).

[21] V.P. Gusynin, V.A. Miransky, and I.A. Shovkovy, Phys. Rev. Lett. 73, 3499 (1994); Phys. Rev. D 52, 4718 (1995).

[22] J. Schwinger, Phys. Rev. 82, 664 (1951).

[23] E.J. Ferrer, V.P. Gusynin, V. de la Incera, preprint cond-mat/0203217

[24] E.V. Gorbar, V.P. Gusynin, V.A. Miransky, I.A. Shovkovy, Phys. Rev. B 66, 045108 (2002).

[25] A. Chodos, K.Everding, and D.A. Owen, Phys. Rev. D 42, 2881 (1990).

[26] R.E. Prange, The Quantum Hall Effect, edited by R.E. Prange and S.M. Girvin, (Springer Verlag, New York, 1987).

[27] G. D. Mahan, Many-Particle Physics, Plenum Press, New York, 1990.

[28] J.S. Langer, Phys. Rev. 127, 5 (1962).

[29] G.M. Eliashberg, Sov. Phys. JETP 14, 886 (1962).

[30] Y. Ando, J. Takeya, Y. Abe, K. Nakamura, and A. Kapitulnik, Phys. Rev. B 62, 626 (2000).

[31] The original Kubo formula requires modification in the case of Hall thermal conductivity [Ref.13 [13] and M. Jonson, S.M. Girvin, Phys. Rev. B 29, 1939 (1984)] in the presence of magnetic field while it remains intact for the longitudinal thermal conductivity studied here.

[32] I.S. Gradshtein and I.M. Ryzhik, Table of Integrals, Series and Products, (Academic Press, Orlando, 1980).

[33] The sum over frequencies appears to be divergent, however, as was shown by V. Ambegaokar and A. Griffin [Phys. Rev. A 137, 1151 (1965)], this divergence results from an improper treatment of time derivatives inside the time-ordered product of heat currents Eq. (5.1). This divergence disappears when the problem is treated more carefully. The prescription is simply to ignore it.

[34] W.V. Liu, Nucl. Phys. B 556, 553 (1999).

[35] http://functions.wolfram.com/ZetaFunctionsandPolylogarithms/ 


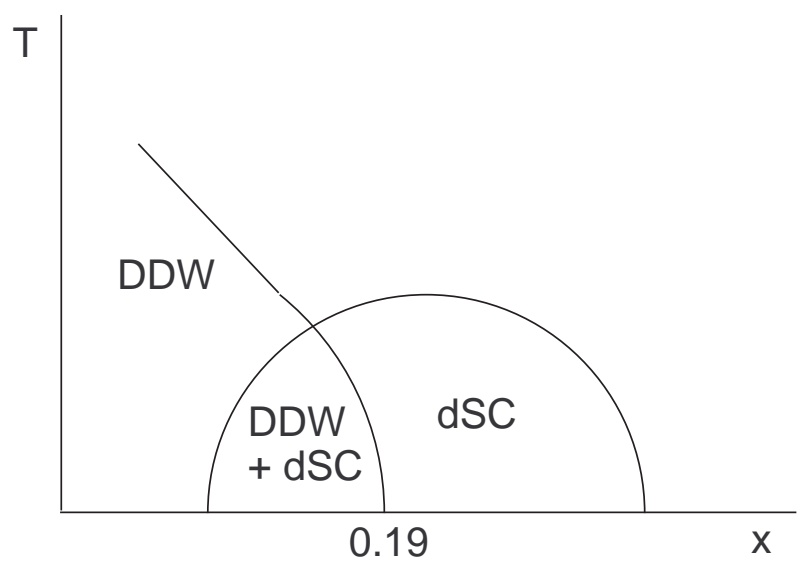

FIG. 1: Schematic phase diagram of cuprates within the DDW scenario.

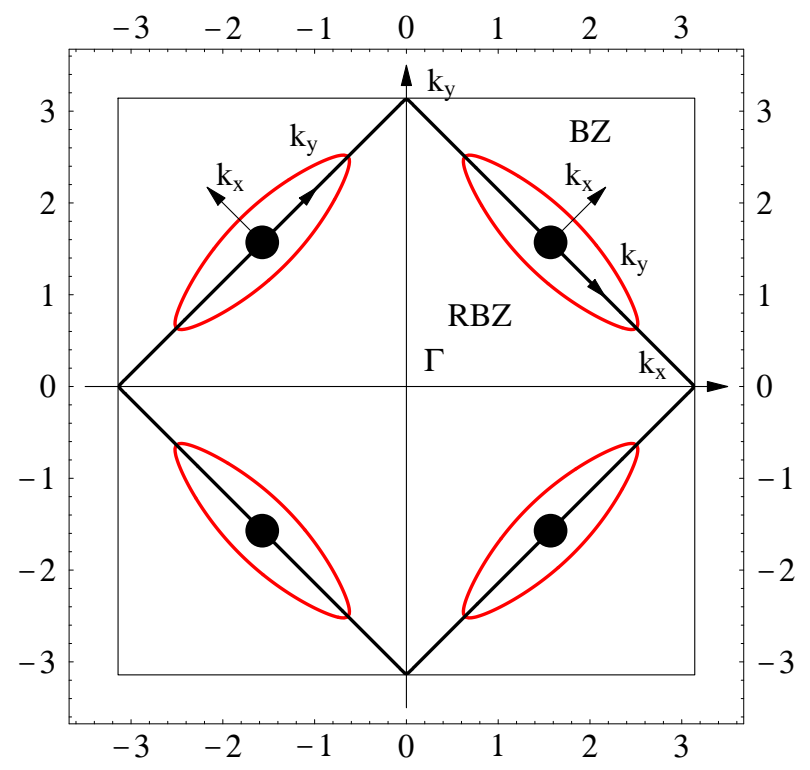

FIG. 2: Fermi surface in the DDW state. The hole pockets are centered around the points $( \pm \pi / 2, \pm \pi / 2)$. We choose the local nodal coordinate systems as shown. 


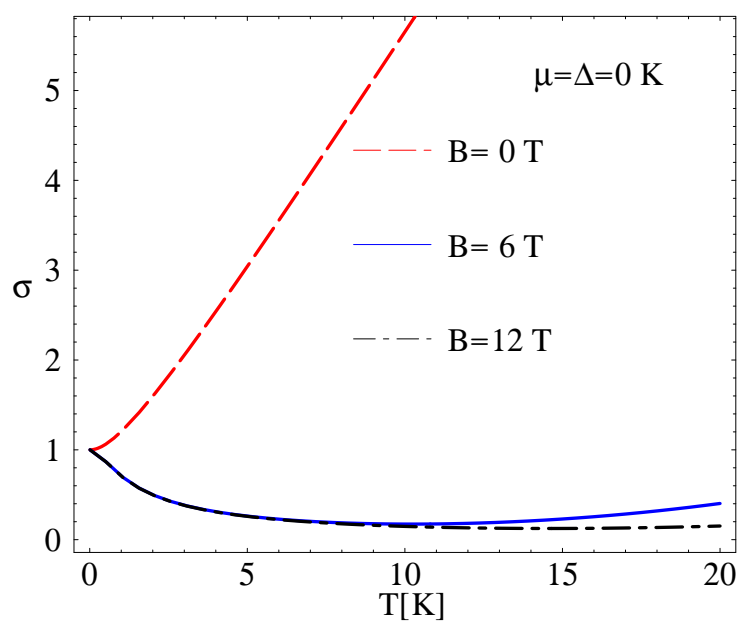

FIG. 3: The normalized conductivity $\sigma / \sigma(\mu=T=B=0)$ as function of temperature, $T$, for three different values of magnetic field $B$ at half-filling, $\mu=0$. The value of $\sigma(\mu=T=B=0)$ is given by Eq. (4.19).

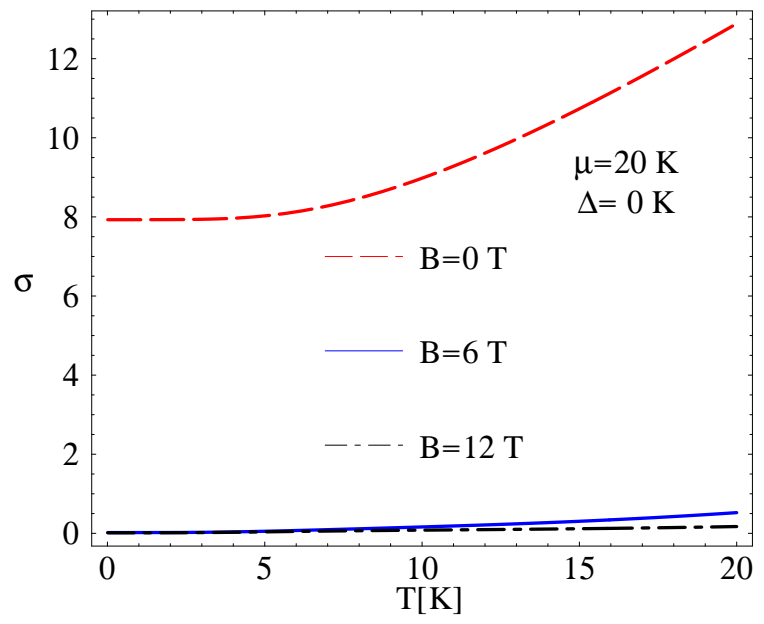

FIG. 4: The normalized conductivity $\sigma / \sigma(\mu=T=B=0)$ as function of temperature, $T$, for three different values of magnetic field $B$ away from half-filling, $\mu=20 \mathrm{~K}$. 


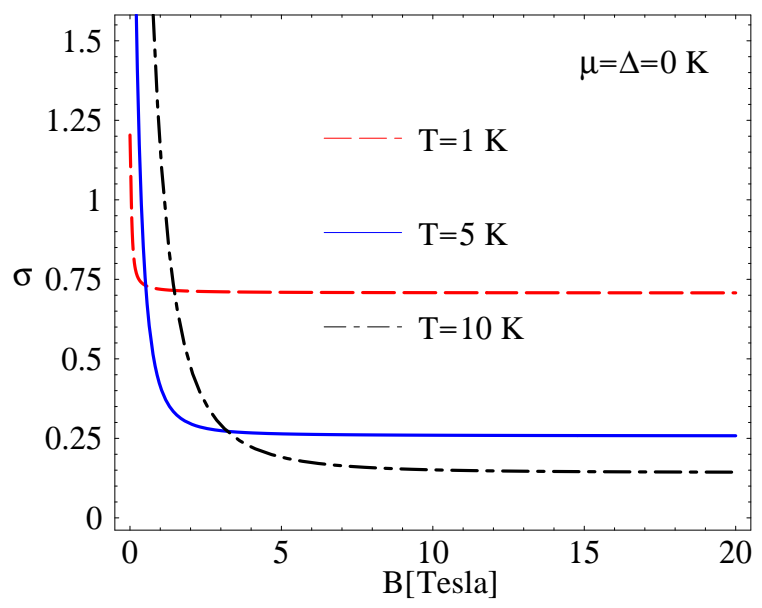

FIG. 5: The normalized conductivity $\sigma / \sigma(\mu=T=B=0)$ as function of field, $B$, for three different values of temperature $T$ at half-filling, $\mu=0$.

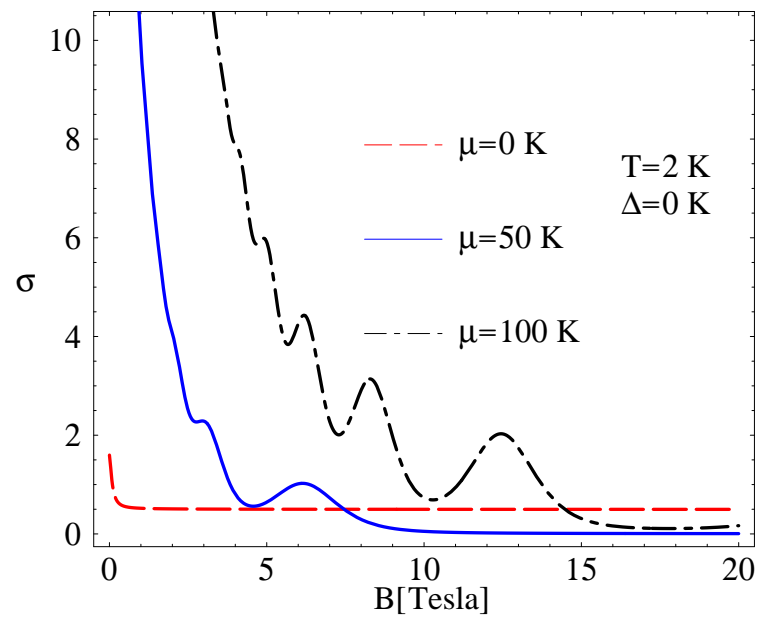

FIG. 6: The normalized conductivity $\sigma / \sigma(\mu=T=B=0)$ as function of field, $B$, for three different values of chemical potential $\mu$ at $T=2 \mathrm{~K}$. 


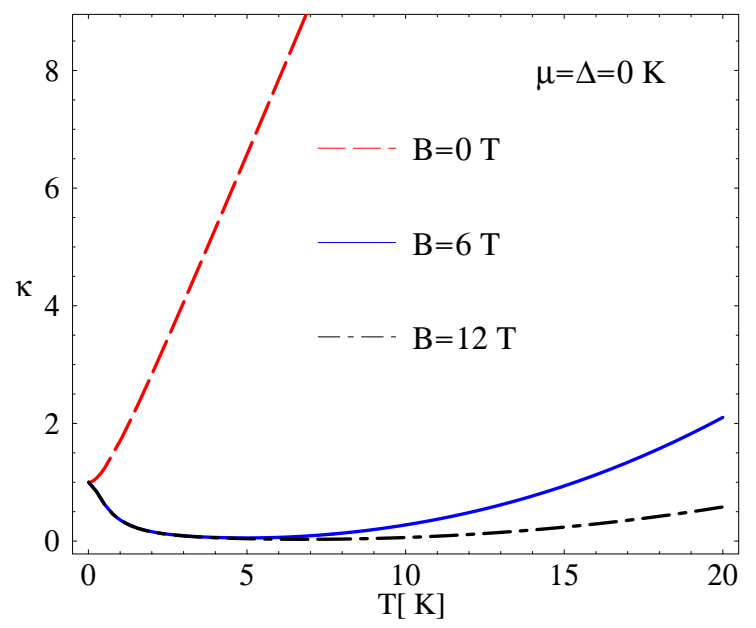

FIG. 7: The normalized thermal conductivity $\kappa / \kappa(\mu, B=0, T \rightarrow 0)$ as function of temperature, $T$, for three different values of magnetic field $B$ at half-filling, $\mu=0$. The value of $\kappa(\mu, B=0, T \rightarrow 0)$ is given by Eq. (5.10).

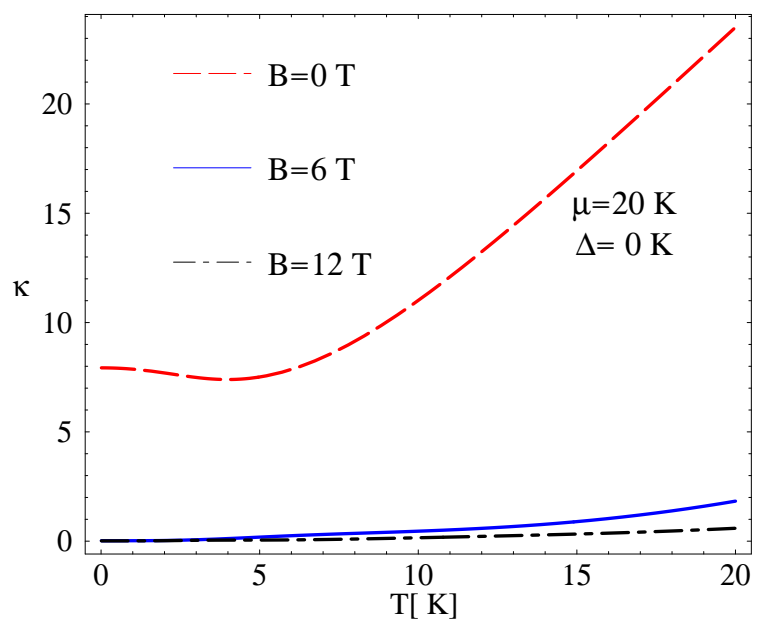

FIG. 8: The normalized thermal conductivity $\kappa / \kappa(\mu, B=0, T \rightarrow 0)$ as function of temperature, $T$, for three different values of magnetic field $B$ away from half-filling, $\mu=20 \mathrm{~K}$. 


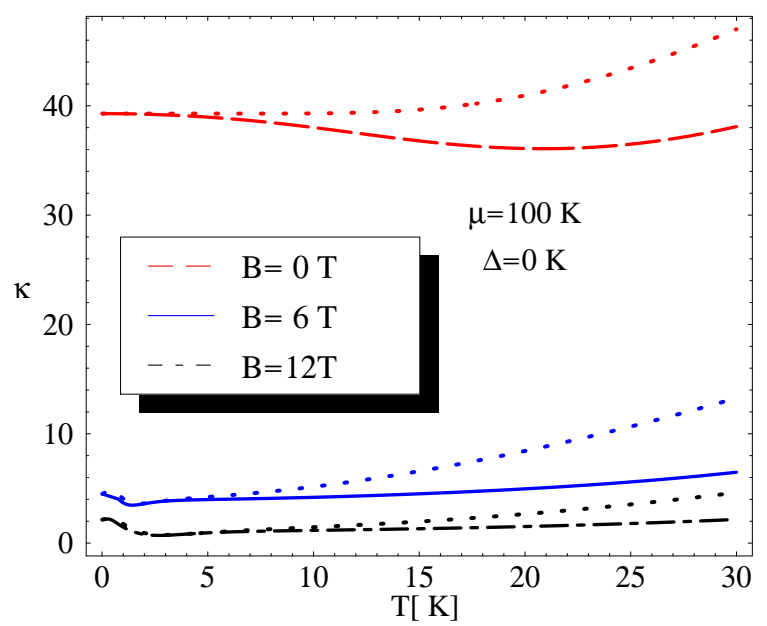

FIG. 9: The normalized thermal conductivity $\kappa / \kappa(\mu, B=0, T \rightarrow 0)$ as function of temperature, $T$, for three different values of magnetic field $B$ away from half-filling, $\mu=100 \mathrm{~K}$. The dotted lines are calculated without the second term of Eq. (5.8) that originates from the condition of absence of the electrical current in the system.

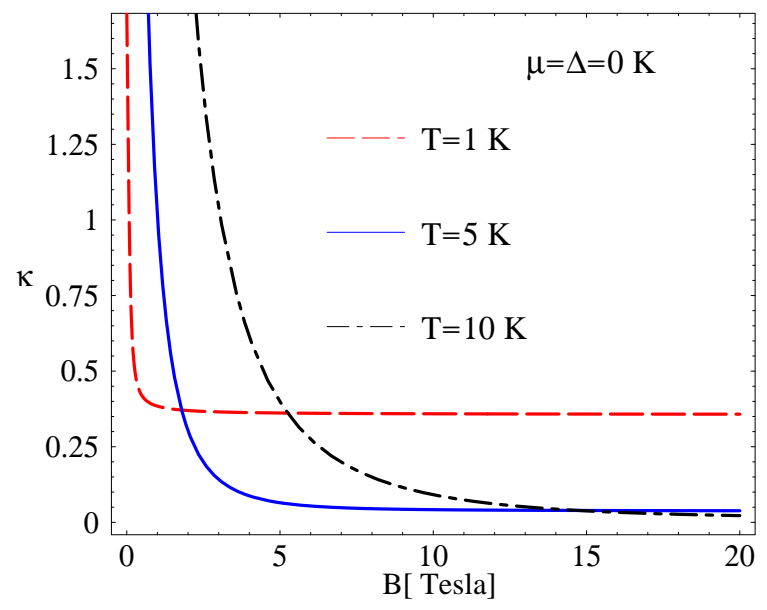

FIG. 10: The normalized thermal conductivity $\kappa / \kappa(\mu, B=0, T \rightarrow 0)$ as function of field, $B$, for three different values of temperature $T$ at half-filling, $\mu=0$. 


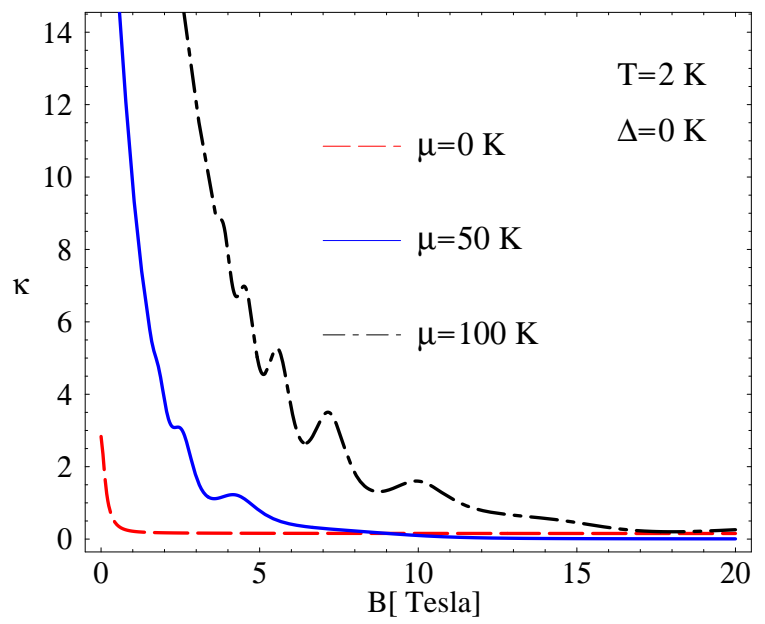

FIG. 11: The normalized thermal conductivity $\kappa / \kappa(\mu, B=0, T \rightarrow 0)$ as function of field, $B$, for three different values of chemical potential $\mu$.

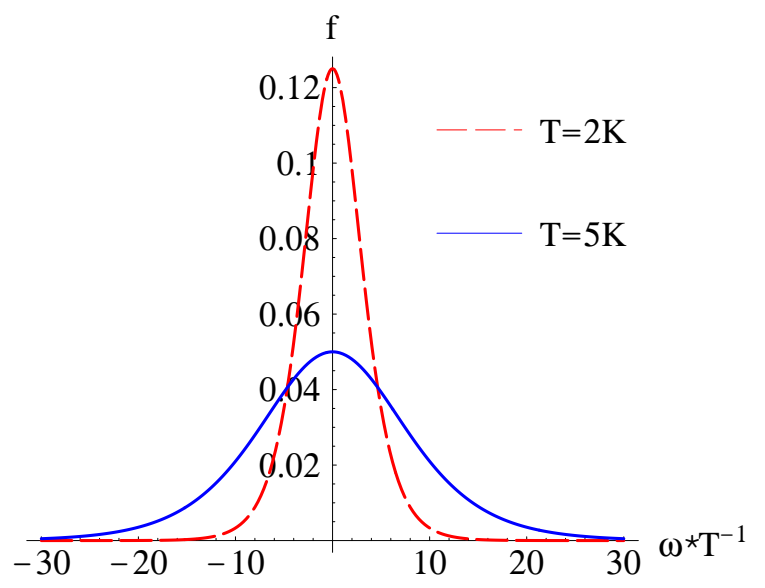

FIG. 12: The expression for electrical conductivity contains the factor $f(\omega)=-n_{F}^{\prime}(\omega-\mu)$ (the case $\mu=0$ is plotted) and thus samples $\mathcal{A}(\omega)$ in the immediate vicinity of the Fermi surface. 


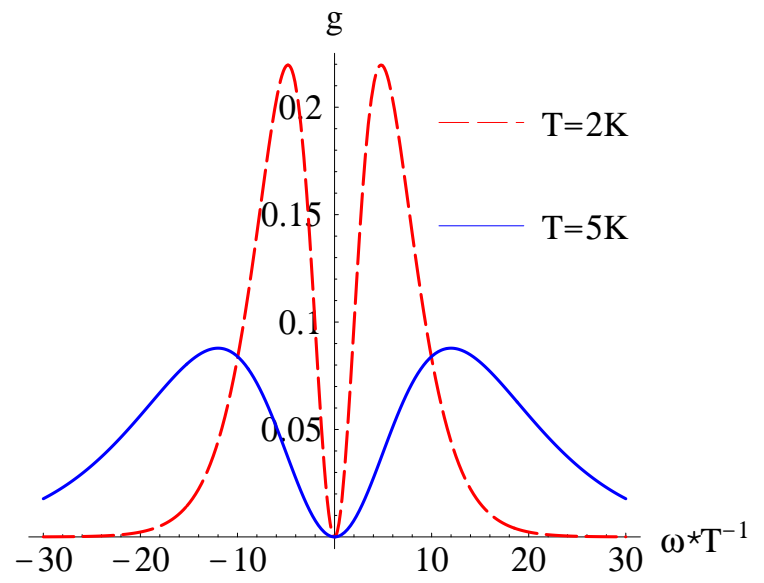

FIG. 13: The expression for thermal conductivity contains the factor $g(\omega)=-n_{F}^{\prime}(\omega-\mu)(\omega-\mu)^{2} / T^{2}$ (the case $\mu=0$ is plotted), and thus measures $\mathcal{A}(\omega)$ in the immediately below and above the Fermi energy.

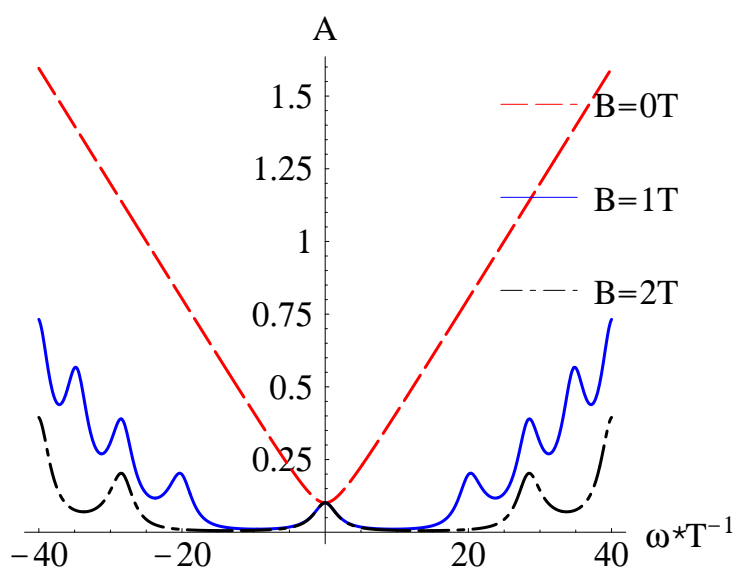

FIG. 14: If $\mathcal{A}(\omega)$ varies appreciably over the energy range from $\mu-k_{B} T$ to $\mu+k_{B} T$ then the Lorenz number deviates from its usual value $L_{0}$.

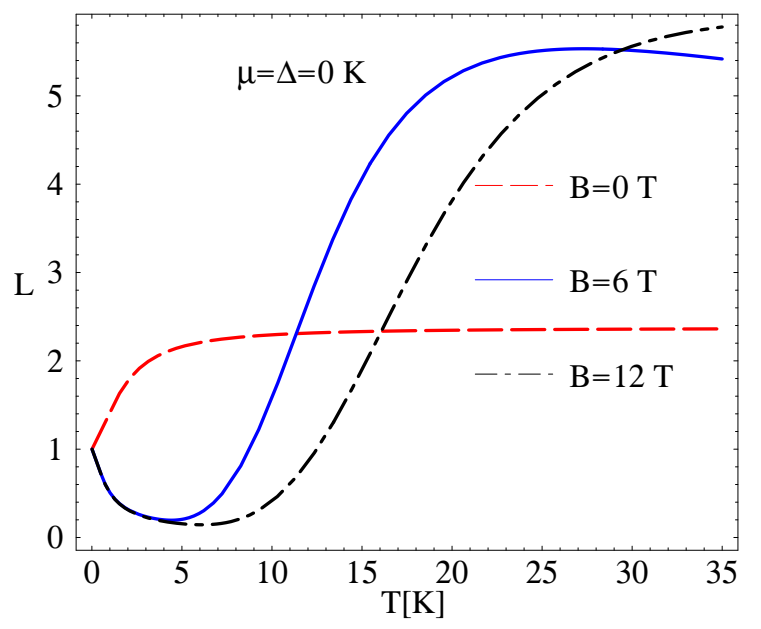

FIG. 15: The normalized Lorenz number $L / L_{0}$ as function of temperature, $T$, for three different values of magnetic field $B$ at half-filling, $\mu=0$. 


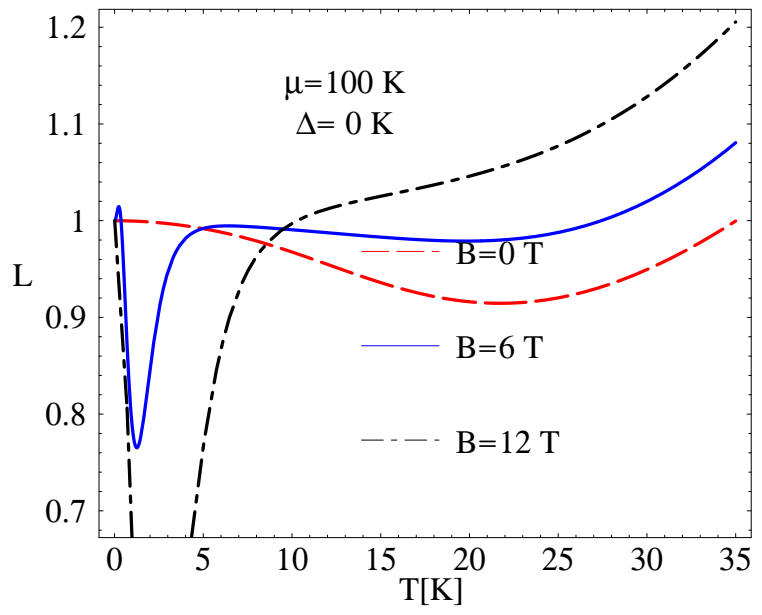

FIG. 16: The normalized Lorenz number $L / L_{0}$ as function of temperature, $T$, for three different values of magnetic field $B$ away from half-filling, $\mu=100 \mathrm{~K}$.

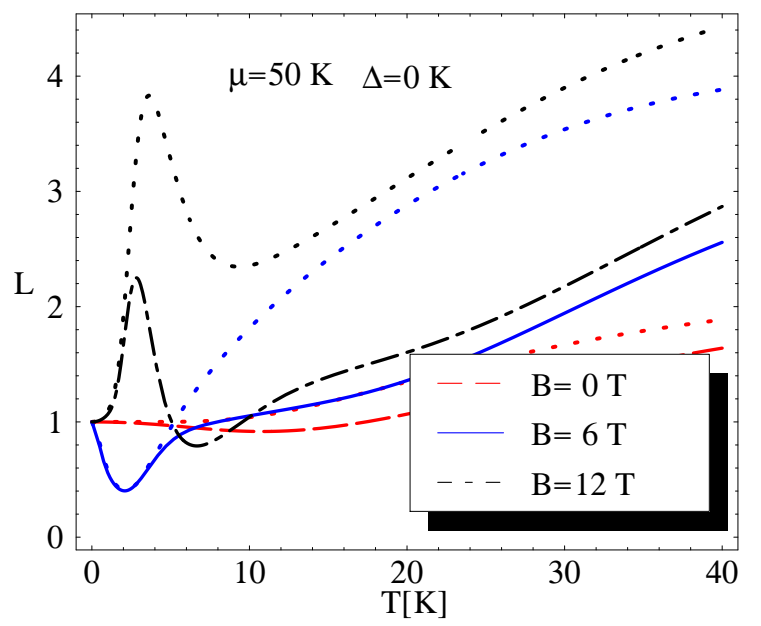

FIG. 17: The normalized Lorenz number $L / L_{0}$ as function of temperature, $T$, for three different values of magnetic field $B$ away from half-filling, $\mu=50 \mathrm{~K}$. The dotted lines are calculated without the second term of Eq. (5.8) that originates from the condition of absence of the electrical current in the system. 